\title{
On the nature of sn stars
}

\section{A detailed abundance study}

\author{
C. Saffe ${ }^{\star}$ and H. Levato ${ }^{\star}$
}

Instituto de Ciencias Astronómicas, de la Tierra y del Espacio (ICATE), C.C 467, 5400 San Juan, Argentina. e-mail: [csaffe,hlevato]@icate-conicet.gob.ar

Received 15 June 2013 / Accepted 2 January 2014

\section{ABSTRACT}

\begin{abstract}
The sn stars were first discoved by Abt \& Levato when studying the spectral types in different open clusters. These stars present sharp Balmer lines, sharp metallic lines (C II, Si II, Ca II, Ti II, Fe II), and broad coreless He I lines. Some of the sn stars seem to be related to CP stars. Initially Abt \& Levato proposed a shell-like nature to explain the sn stars, although this scenario was subsequently questioned. There is no general agreement about their origin. We aim to derive abundances for a sample of 9 stars, including sn and non-sn stars, to determine the possible relation between sn and CP stars and compare their chemical abundances. That most sn stars belong to open clusters allows us to search for a possible relation with fundamental parameters, including the age and rotation. We also study the possible contribution of different effects to the broad He I lines observed in these stars, such as Stark broadening and the possible He-stratification. Effective temperature and gravity were estimated by Strömgren photometry and then refined by requiring ionization and excitation equilibrium of Fe lines. We derived the abundances by fitting the observed spectra with synthetic spectra using an iterative procedure with the SYNTHE and ATLAS9 codes. We derived metallic abundances of 23 different chemical elements for 9 stars and obtained low projected rotational velocities for the sn stars in our sample $\left(v \sin i\right.$ up to $\left.69 \mathrm{~km} \mathrm{~s}^{-1}\right)$. We also compared 5 stars that belong to the same cluster (NGC 6475) and show that the sn characteristics appear in the 3 stars with the lower rotational velocity. However, the apparent preference of sn stars for objects with the lower $v \sin i$ values should be taken with caution due to the small number of objects studied here. We analysed the photospheric chemical composition of sn stars and show that approximately $\sim 40 \%$ of them display chemical peculiarities (such as He-weak and $\mathrm{HgMn}$ stars) within a range of temperature of $10300 \mathrm{~K}-14500 \mathrm{~K}$. However, there are also sn stars with solar or nearly-solar (i.e. non-CP) chemical composition. We have studied the possible contribution of different processes to the broad He I lines present in the sn stars. Although NLTE effects could not be completely ruled out, it seems that NLTE is not directly related to the broad He I profiles observed in the sn stars. The broad-line He I $4026 \AA$ is the clearest example of the sn characteristics in our sample. We succesfully fit this line in 4 out of 7 sn stars by using the appropriate Stark broadening tables, while small differences appear in the other 3 stars. Studying the plots of abundance vs. depth for the He I lines resulted in some sn stars probably being stratified in He. However, a further study of variability in the He I lines would help for determining whether a possible non-uniform He superficial distribution could also play a role in these sn stars. We conclude that the broad He I lines that characterize the sn class could be modelled (at least in some of these stars) by the usual radiative transfer process with Stark broadening, without needing another broadening mechanism. The observed line broadening in sn stars seems to be related to the "normal" He line formation that originates in these atmospheres.
\end{abstract}

Key words. stars: chemically peculiar - stars: abundances - stars: late-type

\section{Introduction}

The "sn" stars were characterized by Abt \& Levato (1977) when classifying the spectral types in the Orion OB1 association. These stars present sharp Balmer line cores, sharp lines of metals (C II, Si II, Ca II, Ti II, Fe II), and broad coreless lines of He I (see also Abt 1978). The authors introduced the "sn" designation because the spectra present at the same time sharp (s) and nebulous (n) lines. Abt (1979) gave a list of 29 sn stars that belong to 12 open clusters and noted that the sn characteristics could occur simultaneously with a variety of chemical peculiarities, including $\mathrm{He}$-weak, $\mathrm{Bp}(\mathrm{Si})$, and $\mathrm{HgMn}$. These chemically peculiar (CP) stars present strong and/or weak intensities in the spectral lines of certain chemical species. The sn characteristics are detected in the spectra of B-type stars (B2-B9) with luminosity classes V-III and in the HR diagram from the ZAMS to well above it. There have been no surveys of field stars for sn spectra.

^ Members of the Carrera del Investigador Científico, CONICET, Consejo Nacional de Investigaciones Científicas y Técnicas de la República Argentina.
Abt \& Levato initially proposed that the effect is probably due to weak shells, generally related to highly rotating stars. Shell stars show both strongly broadened photospheric lines and additional narrow absorption lines (see e.g. the reviews of Porter \& Rivinius 2003; Rivinius et al. 2006). In this context, the sharp lines are formed high in the atmosphere (in the thin shell), while the diffuse or nebulous He I lines are formed lower and show the rapid photospheric rotation. The observed rotational velocity of shell-like stars is usually high, with $v \sin i$ between 150-400 $\mathrm{km} \mathrm{s}^{-1}$ (see e.g. Slettebak 1982; Rivinius et al. 2006). However, the CP stars are usually related to slow rotation. Charbonneau \& Michaud (1988) determined that the maximum rotational velocities allowing the gravitational settling of He are $\sim 75 \mathrm{~km} \mathrm{~s}^{-1}$ for $\mathrm{HgMn}$ stars and $\sim 100 \mathrm{~km} \mathrm{~s}^{-1}$ for FmAm. Once the He abundance has decreased enough in the superficial convection zones, the He convection zone disappears, and then, as the authors explained, the abundance anomalies appear thanks to the radiative diffusion. Observationally, the maximum rotational velocities measured for $\mathrm{HgMn}$ and Am stars are 90 and $120 \mathrm{~km} \mathrm{~s}^{-1}$, respectively (Wolff \& Preston 1978; Abt \& Moyd 1973). More recent works also present similar maximum values 
for CP stars (see e.g. Abt \& Morrel 1995; Abt 2009). Mermilliod (1983) examined sn cluster stars and concluded that their main feature is also a low projected rotational velocity: 12 out of 16 have $v \sin i<50 \mathrm{~km} \mathrm{~s}^{-1}$ and all have $v \sin i<100 \mathrm{~km} \mathrm{~s}^{-1}$. The coexistence of the sn characteristics with chemical peculiarities and the slow rotation, favours a relationship with Bp stars.

Although most of the stars that present the sn characteristics seem to present lower rotational velocities than shell stars, a possible shell-like nature cannot be completely ruled out based solely on the rotational properties. Mermilliod (1983) show that the sn stars with the highest $v \sin i$ in their sample are HD 35502 and HD 36954 (285 and $190 \mathrm{~km} \mathrm{~s}^{-1}$, respectively). These stars belong to the Orion association. Also, Neiner et al. (2005) shows that the star HD 174512 is either a shell Be star or a Herbig $\mathrm{Ae} / \mathrm{Be}$ star, but with a rotational velocity of only $20 \mathrm{~km} \mathrm{~s}^{-1}$. Further studies are needed to confirm the real nature of this interesting object, which is a candidate to be a shell star with a low rotational velocity.

There are several fundamental questions about the group of sn stars. Previous works suggest a high frequency of CP stars among sn stars (see e.g. Table 7 in Abt 1979), which is also supported by their low projected rotational velocity (Mermilliod 1983). One of the aims of this work is to study the possibility that all sn stars were in fact related to CP stars and search for common features in their abundance values. A possible sn-CP relation would indicate that the sn characteristics are present in those atmospheres with the physical conditions required by the diffusion to work efficiently. Also we would like to determine $T_{\text {eff }}$ and $\log g$ for the sn stars. Some of the stars in our sample belong to the same cluster (NGC 6475), so the age and the initial chemical composition are the same for all of them. This allowed us to search for trends with different fundamental parameters. We also included one sn star member of the cluster Melotte 20 and one from M45. Some of these questions are addressed in this work, using a detailed abundance analysis of a sample of sn (and non-sn) stars.

There is no general agreement about the possible contribution of different processes to the broad He I lines observed in the sn stars. For instance, in the temperature range of the sn stars, the Stark effect is an important source of broadening (e.g. Shamey 1969; Barnard et al. 1974). In He-peculiar stars, the atmosphere is possibly He-stratified, and this could affect the profiles of the He I lines $4471 \AA$ A (Farthmann et al. 1994) and $4026 \AA$ (Zboril 2005; Catanzaro 2008). Another aim of this work is to determine the possible contribution of these effects (including NLTE) to the broad He I lines observed in the sn stars. In other words, we study the possibility that the characteristic broadening observed in the He I lines of sn stars could have originated, at least in part, in the atmospheres of these stars.

In addition to the chemical pattern shown by the He-peculiar stars, there are a number of other interesting phenomena present in the circumstellar regions of these stars. Shore \& Adelman (1981) reported variable UV lines in the spectrum of He-rich stars, which are attributed to the magnetic control of the stellar mass outflows in these objects. For the case of He-weak stars, Brown et al. (1984) started a systematic survey of C IV line variations followed by other studies in the literature (Brown et al. 1985; Shore et al. 1987). They studied a total of 15 He-weak stars, including both magnetic and non-magnetic stars, rapid and slow rotators. Within this sample they detected enhanced absorption of C IV only in three He-weak stars (HD 21699, HD 5737, and HD 79158) in which the C IV resonance doublet and the magnetic fields are variable on the rotational time scale. The authors interpret this result with magnetically controlled stellar winds among He-weak stars (Shore et al. 1987; Shore 1987). Notably, these are the only three objects that share the sn designation in their sample based on optical properties. That these three stars display the C IV feature among many He-weak stars at similar temperatures suggest some mechanism working in the sn stars but not in the other "normal" He-weak stars (Shore et al. 1987).

The initial observation of two additional He-weak sn stars (HD 21071 and HD 22136) shows no strong C IV absorption in the spectra (Shore 1986; Shore et al. 1987). However, it is possible that these stars are C IV variable, similar to other Heweak stars, and a single observation corresponds to their weak line phase (Shore 1986). More recently, Shore et al. (2004) have studied the presence of magnetically controlled circumstellar plasmas between the hot members of the He-weak stars, including both sn and non-sn stars. They used UV spectra from the International Ultraviolet Explorer satellite (IUE) which cover the C IV and other important resonance lines. These lines provide a spectroscopic signature of the plasmasphere through the variation in the C IV and Si IV resonance doublets. For the first time, they discovered variable C IV in He-weak stars that do not belong to the sn subclass. The UV line profile variations that are common between He-strong stars (e.g. Shore \& Adelman 1981; Barker et al. 1982) seem to be more widespread among the He-weak stars. In other words, although the UV line profile variation is not rectricted to only the He-weak sn stars, these works show the important result that the He-weak sn stars could present magnetically controlled circumstellar plasmas detected in their UV spectra.

We describe the observational material in Sect. 2. In Sect. 3 we describe the derivation of the temperature and gravity of the sn stars, which are required to obtain an initial model atmosphere for our objects. In Sect. 4 we explain the iterative method applied to derive the abundances by fitting the observed spectra with synthetic ones. We also discuss the possible non-local thermodynamical equilibrium (NLTE) effects present in our calculation. In Sect. 5 we compare the abundance values of sn and CP stars and discuss the possible contribution of different physical processes to the broadening of He I lines. Finally, we conclude the work with some remarks in Sect. 6.

\section{Observational material}

The stellar spectra of the sn stars were obtained from the ESO Science Archive Facility ${ }^{1}$ for the HARPS and UVES spectrographs, and from the Observatoire de Haute-Provence archive for ELODIE spectrograph ${ }^{2}$. HARPS is a fibre-fed highresolution echelle spectrograph (Mayor et al. 2003), installed at the $3.6 \mathrm{~m}$ ESO telescope at La Silla observatory. HARPS resolving power is $\sim 110000$, and it uses two CCD EEV type 44-82 $(4 \mathrm{k} \times 4 \mathrm{k})$ detectors with $15 \mu \mathrm{m}$ pixels. The spectral range covered is $\sim 3800-6800 \AA$. UVES is a two-arm crossdispersed echelle spectrograph (Dekker et al. 2000) attached to the VLT Kueyen UT2 telescope with the Nasmyth B focus. UVES resolving power is $\sim 80000$ and the spectral range covers 3000-5000 ^ (blue) and 4200-11000 A (red). The CCD detector in the blue is an $\operatorname{EEV~}(2 \mathrm{k} \times 4 \mathrm{k})$, while in the red there is an EEV chip mosaic $(2 \mathrm{k} \times 4 \mathrm{k})$ and an MIT/LL chip that has a higher efficiency in the near-infrared. ELODIE is a crossdispersed high-resolution echelle spectrograph attached to the

\footnotetext{
Based on data obtained from the ESO Science Archive Facility, http: //archive.eso.org

2 http://atlas.obs-hp.fr/elodie/
} 
Table 1. Sample of stars studied in this work with spectral types taken from the HIPPARcos database.

\begin{tabular}{lccccc}
\hline \hline $\begin{array}{l}\text { Star } \\
\text { HD }\end{array}$ & Cluster & Instrument & $\begin{array}{c}\text { Spectral } \\
\text { type }\end{array}$ & sn & Ref. \\
\hline 5737 & & HARPS & B7p, He-wk & Y & R3 \\
21071 & Melotte 20 675 & ELODIE & B7V & Y & R1, R2 \\
23950 & M45 3325 & ELODIE & B8, HgMn & Y & R1, R2 \\
74146 & IC 2391 16 & HARPS & B4IV & (Y) & R2 \\
162586 & NGC 6475 56 & HARPS & B8V & Y & R1, R2 \\
162630 & NGC 6475 63 & UVES & B9III & N & R1 \\
162678 & NGC 6475 141 & HARPS & B9V & Y & R1, R2 \\
162679 & NGC 6475 77 & HARPS & B9V & Y & R1, R2 \\
162817 & NGC 6475 110 & UVES & B9.5V & N & R1 \\
\hline
\end{tabular}

References. R1 (Abt 1979), R2 (Mermilliod 1983), R3 (Shore et al. 1987).

Observatoire de Haute-Provence $1.93 \mathrm{~m}$ telescope. The spectral range covers $3850-6800 \AA$ with a resolving power of 42000 , split in 67 spectral orders. This spectrograph uses a $1024 \times 1024$ CCD SITe detector, with $24 \mu \mathrm{m}$ pixels. The signal-to-noise ratio of the spectra varies between 180-370, with an S/N average of $\sim 245$ approximately.

The nine stars in our sample have $V$ magnitudes in the range 4.3-6.2 mag and early-B spectral types. In Table 1 we present some observational data for each star. The spectral types were taken from the HIPPARCos database, while the sn (or non-sn) classification of the stars were taken from the literature (see Cols. 5 and 6). We included 6 sn stars in our sample (Abt 1979; Mermilliod 1983; Shore et al. 1987) and the sn "candidate" HD 74146 (Mermilliod 1983). For comparison purposes, we also added two non-sn stars (HD 162630 and HD 162 817) that belong to NGC 6475 . The reference for the sn classification is presented in the last column.

By inspecting the spectra of our sample stars, the broad line He I $4026 \AA$ is always present and is probably the clearest example of the sn characteristic. We return to this topic in Sect. 5.3.

The spectral lines of the stars were identified using a similar procedure to previous works (e.g. Saffe et al. 2005, 2011). The atomic line list and $\log g f$ data used in this work was basically taken from Fiorella Castelli's linelist ${ }^{3}$, with some changes and additions as described in Castelli \& Hubrig (2004). This linelist includes the Fuhr \& Wiese (2006) $\log g f$ data for several Fe I and Fe II lines, usually referred to as FW06. The line data and $\log g f$ values were updated according to Adelman et al. (2006) and Zavala et al. (2007), in agreement with our previous abundance works (e.g. Saffe et al. 2011).

\section{Atmospheric parameters}

We used the Strömgren uvby $\beta$ mean colours from Hauck \& Mermilliod (1998), together with the calibration of Napiwotzki et al. (1993), to derive a first estimation of $T_{\text {eff }}$ and $\log g$. However, the photometric standard calibrations should be used with caution in the case of CP stars (some of which are included in our sample), owing to their abnormal colours and non-solar chemistry. Adelman \& Rayle (2000) and Netopil et al. (2008) derived $T_{\text {eff }}$ corrections for the Strömgren and other photometric systems, depending on the chemical peculiarity. For the case of HgMn stars, the $T_{\text {eff }}$ directly derived by Strömgren photometry

\footnotetext{
3 http://wwwuser.oat.ts.astro.it/castelli/linelists. html
}

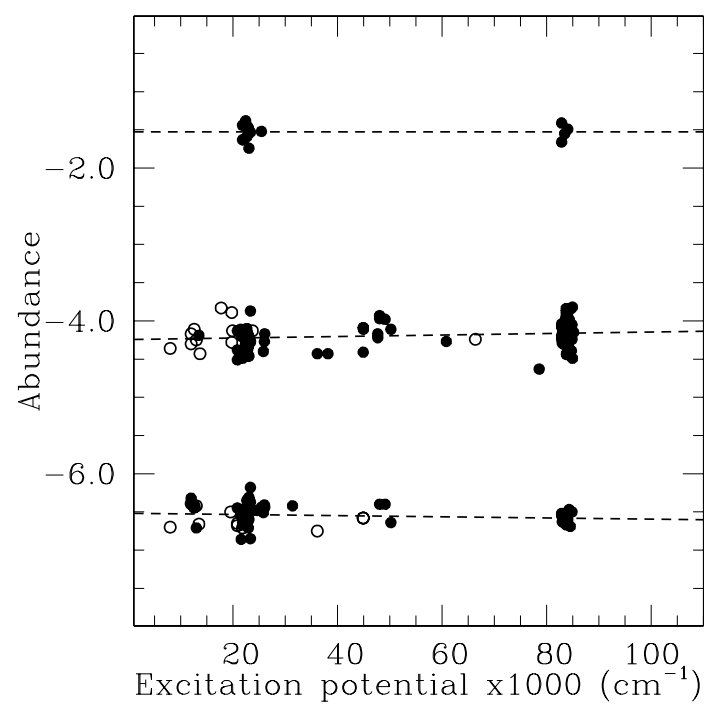

Fig. 1. Example of abundance vs. excitation potential of Fe lines for the stars HD 23950, HD 5737, and HD 162678 (from top to bottom). Empty and filled circles correspond to Fe I and Fe II values, respectively. The straight lines show the position of a linear fit to the data. In this plot the abundance values have been displaced vertically to avoid superposition.

is overestimated, so that a correction is neccessary (Adelman \& Rayle 2000; Netopil et al. 2008). We corrected the derived values of the HgMn star HD 23950 according to Adelman \& Rayle (2000), who found a systematic difference between parameters derived from photometric and spectrophotometric techniques. They suggest a general correction for $\mathrm{HgMn}$ stars that closely agrees with those derived by Netopil et al. (2008). For Am stars, Netopil et al. (2008) conclude that the known calibrations for normal stars can be safely used with high accuracy $(\sim 150 \mathrm{~K})$. Paunzen et al. (2002) show that the standard Strömgren calibration for normal stars is also valid for $\lambda$ Boo stars.

In the case of He-weak stars, the $T_{\text {eff }}$ derived by Strömgren is also overestimated (e.g. Netopil et al. 2008). Leone \& Manfré (1997) determined the temperature and gravity accurately for the He-weak star HD 5737 by using an iterative procedure. They determined the fundamental parameters by fitting the $\mathrm{H} \beta$ line using the program SYNTHE, then they recalculated the He and metal abundances, derived a new ATLAS9 model atmosphere, and restarted the process. In other words, they derived $T_{\text {eff }}$ and $\log g$ but used non-solar abundances. For this object we adopt the accurate parameters obtained by these authors $(13600 \mathrm{~K}$, $3.20 \mathrm{dex})$. The $T_{\text {eff }}$ derived using the Strömgren photometry for Ap stars should also be corrected only if $T_{\text {eff }}>9000 \mathrm{~K}$, while no correction is needed for cooler Ap stars (Netopil et al. 2008). Our sample includes the star HD 162817, which shows mild-Ap rather than classical Ap characteristics (see next sections). The $T_{\text {eff }}$ corrections are not clearly adequate for this object so we prefer to use the original Strömgren values.

We refined the $T_{\text {eff }}$ to achieve the excitation equilibrium condition, i.e. the independence between the abundance values and excitation potential of the lines. In Fig. 1 we present an example of abundance vs. excitation potential of the Fe lines for the stars HD 23950, HD 5737, and HD 162678. The figure also shows a linear fit to the abundance data. Next we adjusted the surface gravity of the sn stars to get ionization equilibrium, i.e. $[\mathrm{Fe} \mathrm{I} / \mathrm{H}]=[\mathrm{Fe} \mathrm{II} / \mathrm{H}]$ within the observational errors. A similar strategy was applied in previous works (e.g. Adelman \& Yuce 2010; Saffe et al. 2011; Saffe 2011). Finally, the adopted values 
Table 2. Fundamental parameters adopted for the stars in our sample.

\begin{tabular}{lccccc}
\hline \hline Star & $\begin{array}{c}T_{\text {eff }} \\
{[\mathrm{K}]}\end{array}$ & $\begin{array}{c}\log g \\
{[\mathrm{dex}]}\end{array}$ & $\begin{array}{c}v \sin i \\
{[\mathrm{~km} / \mathrm{s}]}\end{array}$ & $\begin{array}{c}\text { Age } \\
{[\mathrm{Myr}]}\end{array}$ & Ref. age \\
\hline HD 5737 & 13600 & 3.20 & 17 & & \\
HD 21071 & 14768 & 4.30 & 58 & 90 & $\mathrm{R} 1$ \\
HD 23950 & 12892 & 4.02 & 69 & 100 & $\mathrm{R} 2$ \\
HD 74146 & 14180 & 4.23 & 36 & 53 & $\mathrm{R} 3$ \\
HD 162586 & 12503 & 4.11 & 27 & 224 & $\mathrm{R} 2$ \\
HD 162679 & 10519 & 3.74 & 40 & 224 & $\mathrm{R} 2$ \\
HD 162630 & 10625 & 3.72 & 49 & 224 & $\mathrm{R} 2$ \\
HD 162678 & 10300 & 3.45 & 40 & 224 & $\mathrm{R} 2$ \\
HD 162817 & 10428 & 3.25 & 75 & 224 & $\mathrm{R} 2$ \\
\hline
\end{tabular}

References. For the adopted ages: R1 (Stauffer et al. 1999), R2 (Meynet et al. 1993), R3 (Barrado y Navascués et al. 1999).

are shown in Table 2, together with the corresponding age of the cluster.

To determine the rotational velocities of the sn stars we fitted a synthetic spectrum to $\sim 20 \mathrm{Fe}$ II lines. We used the program SYNTHE (Kurucz \& Avrett 1981), together with the command broaden to reproduce the instrumental broadening of the spectrographs, adopting a resolving power $R$ of 110000,80000 , and 42000 for the HARPS, UVES, and ELODIE spectrographs, respectively. The value of $R$ is approximate, and thus the derived rotational velocities should be taken with caution. The final $v \sin i$ values were obtained using the average of the different Fe lines. The average dispersion is $\sim 0.9 \mathrm{~km} \mathrm{~s}^{-1}$. The rotational velocities are presented in the fourth column of Table 2. We agree with the conclusion of Mermilliod (1983) that the sn stars present low projected rotational velocities.

\section{Abundance analyses}

We used an iterative procedure to determine the abundances of chemical elements in the atmospheres of the sn stars. With the adopted values of $T_{\text {eff }}$ and $\log g$, we started by computing a model atmosphere using the ATLAS9 (Kurucz 1993) code. This initial model has $[\mathrm{M} / \mathrm{H}]=0.0$ i.e. solar metallicity values taken from Grevesse et al. (1996). To determine the abundances we fitted a synthetic spectra to the different lines using the program $\mathrm{SYNTHE}^{4}$. With the new abundance values, we derived a new model atmosphere and started the process again.

We compared the synthetic and observed spectra numerically using the $\chi^{2}$ function (the cuadratic sum of the differences between both spectra). The abundance is modified in steps of 0.01 dex for each spectral line, until a minimum of $\chi^{2}$ is obtained. We present the abundances for each sn star studied in this work in Tables 3-5. In the ATLAS9 model atmospheres, for each chemical species the abundance is directly given as $\log \left(N / N_{T}\right)$, where $N$ is the number of absorbing atoms per unit volume and $N_{T}$ the number of particles of both $\mathrm{H}$ and $\mathrm{He}$. In other words, it is only necessary to specify the ratio $N / N_{T}$ to determine certain abundance value. The final values presented in Tables 3-5 correspond to the average and rms $(\sigma)$ derived from different spectral lines. To show the possible overabundances or underabundances of the sn stars, we also present the average using the square bracket notation, which denotes abundances relative to the Sun, i.e. $\left[N / N_{T}\right]=\log \left(N / N_{T}\right)_{S \operatorname{Star}}-\log \left(N / N_{T}\right)_{\text {Sun }}$. Solar abundance values $\log \left(N / N_{T}\right)_{\text {Sun }}$ have been taken from Grevesse et al. (1996). We caution that the error of the abundances $\sigma$ presented in these tables correspond to the dispersion

\footnotetext{
4 http://kurucz.harvard.edu/programs.html
}

of the abundances derived from different lines and do not include the error in the abundances of the Sun, in order to properly compare with previous abundance works (e.g. Zavala et al. 2007; Saffe et al. 2011). No uncertainty is quoted when only one line is present.

In Table 6 we show a sample of the line by line abundances, in this case for the star HD 162586. The columns present the wavelength and abundance $\log \left(N / N_{T}\right)$ of the line, $\log g f$ with their corresponding reference, the minimum $\chi^{2}$ derived, and the residual intensity of the isolated synthetic line (before rotational and instrumental broadening). The next column presents a flag ( $\mathrm{Y}$ or $\mathrm{N}$ ) if the line is used or not to calculate the average abundance of the element, and finally a comment for each particular line. The chemical species are identified at the beginning of the line lists using the code " $z . l$ ", where $z$ is the atomic number and $i$ the ionization stage. For instance, 14.01 corresponds to $\mathrm{Si}$ II. The same code, $z . i$, is used to identify elements in most figures in this work. There is an on-line version of the complete Table 6 including a plot for each line $e^{5}$.

Figure 2 shows two examples of synthetic fits of the same spectral region near the line Ni II 3849.55 for the stars HD 162586 and HD 162630 (upper and lower panels, respectively). The synthethic lines are identified in the figure using the notation "Lambda Code Int", i.e. the wavelength of the line in $\AA$, the code $z . i$ of the element and the relative intensity of the line. For instance, 3849.5528 .010 .3917 correspond to the line $3849.55 \AA$ of Ni II, with a relative intensity of 0.3917 . The two thin red curves correspond to $\pm 1 \sigma$ abundance variation in the synthethic spectra, where $\sigma$ is the standard deviation derived in this case from different Ni II lines. Both the $\pm 1 \sigma$ abundance curves and the notation for identifying the synthethic lines are used in most figures of this work. In the upper panel, the lines Mg II $3848 \AA$, Ni II $3849 \AA$, and Mg II $3950 \AA$ are present (with some blends), while in the lower panel a higher $v \sin i$ smooths the region and strongly blends the lines. In both cases the synthetic spectra fit well the observed spectra.

In Fig. 3 we show two plots corresponding to the spectral region near the line Si II 4076, for HD 162586 and HD 5737 (upper and lower panels, respectively). In the upper panel there are three intense lines (Si II $4075 \AA$, $4076 \AA$, and Ni II $4077 \AA$ ) while in the lower panel there are also C II lines (such as $4074.8 \AA$ and $4075.8 \AA$ ) blended with other less intense lines. We see that the use of synthetic spectra allows the modelling of spectral lines even for rotating stars where some blends are present.

The uncertainty in the derived abundance values have different sources. The effective temperature and gravity were first estimated by using the Strömgren photometry and corrected for CP stars. This estimation presents average dispersions of $\sim 200 \mathrm{~K}$ and $\sim 0.2$ dex for $T_{\text {eff }}$ and $\log g$, respectively (Adelman \& Rayle 2000; Netopil et al. 2008). We estimate similar dispersion values for $T_{\text {eff }}$ and $\log g$ by achieving the excitation and ionization equilibrium. The total dispersion from the combination of photometry and equilibrium conditions (assuming that they are independent) amount to $\sim 300 \mathrm{~K}$ in $T_{\text {eff }}$ and $\sim 0.3$ dex in $\log g$, approximately. We estimate that the uncertainty in $\log g f$ values may cause average differences of about 0.09 dex $(\sim 4.7 \%)$ in the calculated metallicity. Finally, to provide an estimation of the "typical" uncertainty and give an idea of the sensitivity of our results, raising the temperature of the sn sample $300 \mathrm{~K}$ $(\sim 3 \%)$ increases the average abundance by $\sim 0.07 \operatorname{dex}(3.2 \%)$,

5 http://icate-conicet.gob.ar/saffe/sn/Html/Salida7. html 
Table 3. Derived abundances for the stars in our sample.

\begin{tabular}{|c|c|c|c|c|c|c|c|c|c|c|}
\hline Species & & $\begin{array}{l}\text { HD } \\
5737\end{array}$ & & & $\begin{array}{c}\text { HD } \\
21071 \\
\end{array}$ & & & $\begin{array}{c}\mathrm{HD} \\
23950\end{array}$ & & Sun \\
\hline & $\log \left(N / N_{T}\right)$ & {$\left[N / N_{T}\right]$} & $\sigma(n)$ & $\log \left(N / N_{T}\right)$ & {$\left[N / N_{T}\right]$} & $\sigma(n)$ & $\log \left(N / N_{T}\right)$ & {$\left[N / N_{T}\right]$} & $\sigma(n)$ & $\log \left(N / N_{T}\right)_{\text {Sun }}$ \\
\hline $\mathrm{He} \mathrm{I}$ & -1.75 & -0.68 & $0.16(7)$ & -1.18 & -0.11 & $0.06(9)$ & -2.04 & -0.97 & $0.02(4)$ & -1.07 \\
\hline C II & -3.48 & 0.04 & $0.20(6)$ & -3.91 & -0.39 & (1) & -3.96 & -0.44 & (1) & -3.52 \\
\hline $\mathrm{Mg}$ II & -4.6 & -0.14 & $0.10(5)$ & -4.59 & -0.13 & $0.06(4)$ & -4.99 & -0.53 & $0.10(2)$ & -4.46 \\
\hline Al I & & & & & & & -4.93 & 0.64 & (1) & -5.57 \\
\hline Al II & -6.95 & -1.38 & (1) & -5.79 & -0.22 & (1) & & & & -5.57 \\
\hline Si II & -4.65 & -0.16 & $0.19(12)$ & -4.72 & -0.23 & $0.05(3)$ & -4.83 & -0.34 & $0.07(5)$ & -4.49 \\
\hline P II & -6.57 & 0.02 & $0.25(2)$ & & & & -5.4 & 1.19 & $0.32(2)$ & -6.59 \\
\hline S II & -5.1 & -0.39 & $0.15(20)$ & -5.12 & -0.41 & $0.12(2)$ & & & & -4.71 \\
\hline $\mathrm{Cl}$ II & -5.21 & 1.33 & $0.23(8)$ & & & & & & & -6.54 \\
\hline $\mathrm{Ca}$ II & -5.17 & 0.51 & (1) & & & & -5.4 & 0.28 & (1) & -5.68 \\
\hline Sc II & -7.71 & 1.16 & $0.15(2)$ & & & & & & & -8.87 \\
\hline Ti II & -5.78 & 1.24 & $0.19(50)$ & & & & -6.21 & 0.81 & $0.22(14)$ & -7.02 \\
\hline Cr II & -5.68 & 0.69 & $0.13(35)$ & & & & -5.85 & 0.52 & $0.23(5)$ & -6.37 \\
\hline Mn I & & & & & & & -3.98 & 2.67 & 0.17 (3) & -6.65 \\
\hline Mn II & & & & & & & -4.49 & 2.16 & $0.22(20)$ & -6.65 \\
\hline $\mathrm{Fe} I$ & -4.13 & 0.41 & 0.15 (13) & -4.74 & -0.2 & (1) & & & & -4.54 \\
\hline Fe II & -4.2 & 0.34 & $0.13(106)$ & -4.74 & -0.2 & $0.15(14)$ & -4.4 & 0.14 & $0.26(20)$ & -4.54 \\
\hline $\mathrm{Ni}$ II & -6.73 & -0.94 & $0.13(2)$ & & & & & & & -5.79 \\
\hline Ga II & & & & & & & -5.53 & 3.63 & $0.17(3)$ & -9.16 \\
\hline Sr II & -7.03 & 2.04 & $0.16(3)$ & & & & & & & -9.07 \\
\hline $\mathrm{Nd}$ II & -8.98 & 1.56 & 0.18 (4) & & & & & & & -10.54 \\
\hline $\mathrm{Hg}$ II & & & & & & & -5.73 & 5.18 & (1) & -10.91 \\
\hline
\end{tabular}

Notes. For each species, we present the abundance $\log \left(N / N_{T}\right)$, the abundance relative to the Sun, $\left[N / N_{T}\right]$, and the standard deviation $\sigma$ with the number of lines used $(n)$. The last column presents the solar abundances $\log \left(N / N_{T}\right)_{\text {Sun }}$ taken from Grevesse et al. (1996).

Table 4. Derived abundances for the stars in our sample.

\begin{tabular}{|c|c|c|c|c|c|c|c|c|c|c|}
\hline Species & & $\begin{array}{c}\text { HD } \\
74146\end{array}$ & & & $\begin{array}{c}\text { HD } \\
162586 \\
\end{array}$ & & & $\begin{array}{c}\text { HD } \\
162630 \\
\end{array}$ & & Sun \\
\hline & $\log \left(N / N_{T}\right)$ & {$\left[N / N_{T}\right]$} & $\sigma(n)$ & $\log \left(N / N_{T}\right)$ & {$\left[N / N_{T}\right]$} & $\sigma(n)$ & $\log \left(N / N_{T}\right)$ & {$\left[N / N_{T}\right]$} & $\sigma(n)$ & $\log \left(N / N_{T}\right)_{\text {Sun }}$ \\
\hline $\mathrm{He} \mathrm{I}$ & -0.78 & 0.29 & $0.07(9)$ & -0.78 & 0.29 & $0.05(8)$ & -1.23 & -0.16 & $0.08(5)$ & -1.07 \\
\hline C II & -3.44 & 0.08 & $0.24(5)$ & -3.27 & 0.25 & $0.34(5)$ & -3.5 & 0.02 & (2) & -3.52 \\
\hline N II & & & & -3.69 & 0.43 & (1) & & & & -4.12 \\
\hline O I & & & & -3.5 & -0.29 & $0.01(3)$ & & & & -3.21 \\
\hline $\mathrm{Mg} \mathrm{I}$ & & & & -4.07 & 0.39 & $0.14(4)$ & & & & -4.46 \\
\hline $\mathrm{Mg}$ II & -4.87 & -0.41 & $0.12(4)$ & -4.55 & -0.09 & $0.06(6)$ & -4.36 & 0.1 & $0.06(2)$ & -4.46 \\
\hline $\mathrm{Al} \mathrm{I}$ & & & & & & & -5.56 & 0.01 & (1) & -5.57 \\
\hline $\mathrm{Al}$ II & -6.2 & -0.63 & (1) & -5.69 & -0.12 & (1) & -5.26 & 0.31 & (1) & -5.57 \\
\hline Si II & -4.92 & -0.43 & $0.17(6)$ & -4.59 & -0.09 & $0.05(9)$ & -4.42 & 0.07 & $0.08(5)$ & -4.49 \\
\hline S II & -4.68 & 0.03 & $0.18(10)$ & -4.66 & 0.05 & $0.07(7)$ & & & & -4.71 \\
\hline $\mathrm{Ca}$ II & -6.45 & -0.77 & (1) & -5.59 & 0.09 & (1) & -5.66 & 0.02 & (1) & -5.68 \\
\hline Sc II & & & & -9.02 & -0.15 & (1) & & & & -8.87 \\
\hline Ti II & & & & -6.84 & 0.18 & $0.21(20)$ & -6.98 & 0.04 & $0.18(12)$ & -7.02 \\
\hline Cr II & & & & -6.25 & 0.12 & $0.11(16)$ & -6.23 & 0.14 & $0.24(8)$ & -6.37 \\
\hline $\mathrm{Fe} \mathrm{I}$ & & & & -4.41 & 0.13 & $0.12(15)$ & -4.44 & 0.1 & $0.14(7)$ & -4.54 \\
\hline $\mathrm{Fe}$ II & -5.22 & -0.68 & $0.22(13)$ & -4.57 & -0.03 & $0.14(60)$ & -4.41 & 0.13 & $0.11(16)$ & -4.54 \\
\hline Ni II & & & & -5.79 & 0 & $0.15(3)$ & -5.41 & 0.38 & $0.29(3)$ & -5.79 \\
\hline Sr II & & & & -8.66 & 0.41 & $0.15(2)$ & -8.32 & 0.75 & $0.14(2)$ & -9.07 \\
\hline
\end{tabular}

Notes. We use the same notation as in Table 3.

and raising the surface gravity by $0.3 \mathrm{dex}(\sim 21 \%)$ increases the average abundance by $\sim 0.01 \operatorname{dex}(<1 \%)$.

\subsection{NLTE effects}

Most of the lines analysed here are weak lines formed deep in the atmosphere where LTE should prevail. They are well-suited to abundance determinations. We have also included data for hyperfine splitting for the selected transitions when relevant, using the linelist gfhyperall.dat ${ }^{6}$. However, the smearing out of spectra by stellar rotation clearly prevents us from detecting signatures of hyperfine splitting and isotopic shifts.

Departures from LTE are more pronounced in stars with high temperature and with low gravity and metallicity. For instance, the reduction of surface gravity results in a decreased efficiency of collisions with electrons and hydrogen atoms, reducing the thermalizing effect, which leads to stronger NLTE effects

6 http://kurucz . harvard.edu/LINELISTS/GFHYPERALL/ 
Table 5. Derived abundances for the stars in our sample.

\begin{tabular}{|c|c|c|c|c|c|c|c|c|c|c|}
\hline Species & & $\begin{array}{c}\text { HD } \\
162678\end{array}$ & & & $\begin{array}{c}\text { HD } \\
162679\end{array}$ & & & $\begin{array}{c}\text { HD } \\
162817\end{array}$ & & Sun \\
\hline & $\log \left(N / N_{T}\right)$ & {$\left[N / N_{T}\right]$} & $\sigma(n)$ & $\log \left(N / N_{T}\right)$ & {$\left[N / N_{T}\right]$} & $\sigma(n)$ & $\log \left(N / N_{T}\right)$ & {$\left[N / N_{T}\right]$} & $\sigma(n)$ & $\log \left(N / N_{T}\right)_{\text {Sun }}$ \\
\hline $\mathrm{He} \mathrm{I}$ & -1.2 & -0.13 & $0.11(8)$ & -1.2 & -0.13 & $0.05(7)$ & -1.43 & -0.36 & (1) & -1.07 \\
\hline C II & -3.9 & -0.38 & 0.08 (4) & -3.97 & -0.45 & $0.07(3)$ & & & & -3.52 \\
\hline O I & -3.52 & -0.31 & $0.02(2)$ & -3.49 & -0.28 & $0.01(3)$ & & & & -3.21 \\
\hline $\mathrm{Mg} \mathrm{I}$ & -4.12 & 0.34 & 0.17 (3) & & & & -3.57 & 0.89 & $0.23(2)$ & -4.46 \\
\hline Mg II & -4.53 & -0.07 & $0.12(5)$ & -4.51 & -0.05 & $0.09(5)$ & -4.21 & 0.25 & $0.04(2)$ & -4.46 \\
\hline $\mathrm{Al} \mathrm{I}$ & -5.85 & -0.28 & (1) & -5.74 & -0.17 & (1) & & & & -5.57 \\
\hline $\mathrm{Al}$ II & -5.65 & -0.08 & (1) & -5.63 & -0.06 & (1) & -5.68 & -0.11 & (1) & -5.57 \\
\hline Si II & -4.54 & -0.05 & $0.06(7)$ & -4.56 & -0.07 & $0.08(8)$ & -4.49 & 0 & $0.09(6)$ & -4.49 \\
\hline Ca II & -5.66 & 0.02 & (1) & -5.6 & 0.08 & (1) & -5.13 & 0.55 & (1) & -5.68 \\
\hline Sc II & -9.16 & -0.29 & $0.06(2)$ & -9.32 & -0.45 & $0.20(2)$ & -8.68 & 0.19 & (1) & -8.87 \\
\hline Ti II & -6.87 & 0.15 & $0.23(31)$ & -6.74 & 0.28 & $0.23(34)$ & -6.35 & 0.67 & $0.23(21)$ & -7.02 \\
\hline Cr II & -6.28 & 0.09 & $0.16(23)$ & -6.15 & 0.22 & $0.23(26)$ & -6.13 & 0.24 & $0.20(12)$ & -6.37 \\
\hline $\mathrm{Fe} \mathrm{I}$ & -4.49 & 0.05 & $0.11(14)$ & -4.39 & 0.15 & $0.16(24)$ & -4.05 & 0.49 & $0.25(11)$ & -4.54 \\
\hline Fe II & -4.56 & -0.02 & $0.14(49)$ & -4.44 & 0.1 & $0.17(53)$ & -4.25 & 0.29 & 0.19 (19) & -4.54 \\
\hline Ni II & -5.92 & -0.13 & $0.01(2)$ & -5.9 & -0.11 & $0.20(2)$ & -5.51 & 0.28 & $0.38(2)$ & -5.79 \\
\hline Sr II & -9.2 & -0.13 & 0.01 (2) & -9.12 & -0.05 & $0.02(2)$ & -8.17 & 0.9 & (1) & -9.07 \\
\hline
\end{tabular}

Notes. We use the same notation as in Table 3.

Table 6. Sample of the line by line abundance table.

\begin{tabular}{|c|c|c|c|c|c|c|c|}
\hline Lambda & Abund & $\log g f$ & Ref & $\chi^{2}$ & Int & AbAccept & Comment \\
\hline \multicolumn{8}{|l|}{$14.01 \mathrm{Si} \mathrm{II}$} \\
\hline 3853.66 & -4.50 & -1.440 & LA & 0.002441 & 0.3534 & Y & \\
\hline 3856.02 & -4.57 & -0.490 & LA & 0.006904 & 0.2570 & Y & \\
\hline 3862.60 & -4.60 & -0.740 & LA & 0.004232 & 0.2811 & Y & \\
\hline 4075.45 & -4.63 & -1.400 & SG & 0.001527 & 0.8158 & Y & \\
\hline 4076.78 & -4.59 & -1.670 & SG & 0.000888 & 0.8748 & Y & \\
\hline 4128.05 & -4.58 & 0.380 & LA & 0.004738 & 0.3695 & Y & \\
\hline 4130.89 & -4.60 & 0.530 & LA & 0.002375 & 0.3549 & Y & \multirow{3}{*}{ Weak and blended with Fe I 4673.2} \\
\hline 4673.28 & -4.48 & -0.710 & KX & 0.001259 & 0.9896 & Y & \\
\hline 5041.02 & -4.64 & 0.290 & SG & 0.001421 & 0.5269 & Y & \\
\hline Si II Avg & -4.59 & $+/-0.05(9)$ & & & & & \\
\hline \multicolumn{8}{|l|}{$16.01 \mathrm{~S}$ II } \\
\hline 4153.07 & -4.71 & 0.620 & WS & 0.001254 & 0.8554 & Y & \\
\hline 4162.66 & -4.68 & 0.780 & WS & 0.001783 & 0.8287 & Y & \multirow{7}{*}{ No fit } \\
\hline 4415.34 & -2.97 & -1.080 & KP & 0.006496 & 0.8903 & $\mathrm{~N}$ & \\
\hline 4991.97 & -4.60 & -0.650 & WS & 0.000846 & 0.9455 & Y & \\
\hline 5014.04 & -4.68 & 0.030 & $\mathrm{KX}$ & 0.002286 & 0.9011 & Y & \\
\hline 5032.43 & -4.61 & 0.180 & WS & 0.000620 & 0.8455 & Y & \\
\hline 5212.62 & -4.77 & 0.240 & WS & 0.001127 & 0.9439 & Y & \\
\hline 5320.72 & -4.56 & 0.460 & WS & 0.001090 & 0.9052 & $\mathrm{Y}$ & \\
\hline S II Avg & -4.66 & $+/-0.07(7)$ & & & & & \\
\hline \multicolumn{8}{|l|}{$20.01 \mathrm{Ca}$ II } \\
\hline 3933.66 & -5.59 & 0.130 & WM & 0.019829 & 0.1294 & $\mathrm{Y}$ & Intense line \\
\hline Ca II Avg & -5.59 & (1) & & & & & \\
\hline
\end{tabular}

Notes. The chemical species are identified at the beginning of the line lists using the code " $z . i$ " where $z$ is the atomic number and $i$ the ionization stage. For instance, 14.01 corresponds to Si II. The same code $z . i$ is used to identify elements in the figures of this work.

(see e.g. Gratton et al. 1999). It is not an obvious task to disentangle non-LTE effects from other sources of uncertainty in abundance analyses. Subsequently, some particular transitions should be taken with caution. In the line-by-line abundance table, we give a warning of "Possible NLTE effects" for those lines where we suspect the presence of these effects. The case of possible NLTE effects, particularly for the He I lines, is considered in the next sections (see Sect. 6.1).

For instance, for C II both weak and intense lines show pronounced NLTE effects (Przybilla et al. 2011) except for C II $5145 \AA$ and the other members of the multiplet. It is possible to detect NLTE effects by comparing the abundance values derived from C II 5145 (which is not affected by NLTE) and abundances from intense C II lines (which present NLTE effects). For O I, NLTE effects are expected, especially in the nearIR triplet O I $7771 \AA$ and the other lines of the same multiplet (see e.g. Sitnova et al. 2013; Przybilla et al. 2011). Przybilla et al. (2001) evaluated the NLTE line formation of neutral and singlyionized $\mathrm{Mg}$, and concluded that the NLTE effects for Mg II are usually small except for the intense line Mg II $4481 \AA$ A. This line systematically yields higher abundance values than the other $\mathrm{Mg}$ II lines, between $0.2-0.8 \mathrm{dex}$ for stars with $T_{\text {eff }}$ in the range $9100-9550 \mathrm{~K}$. Most of the Si lines are also affected in stars with $T_{\text {eff }}$ higher than $15000 \mathrm{~K}$ (e.g. Przybilla et al. 2011). 
C. Saffe and H. Levato: On the nature of sn stars. I.
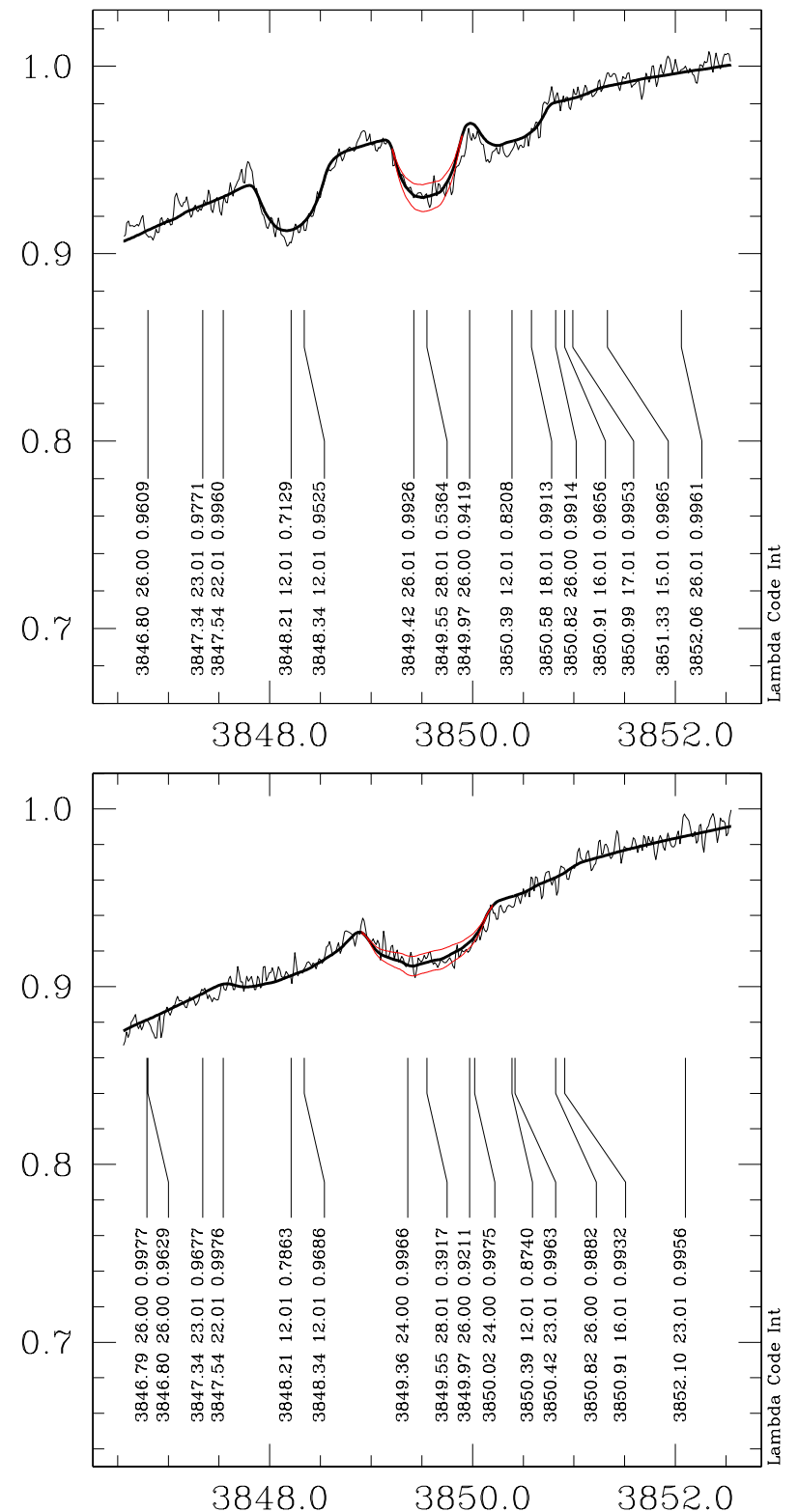

Fig. 2. Comparison of the spectral region near the Ni II 3849 line for HD 162586 (upper panel) and HD 162630 (lower panel). The observed and synthetic spectra are shown by thin and thick lines, respectively. The 2 thin red curves correspond to $\pm 1 \sigma$ abundance variation of the synthethic spectra.

\section{Discussion}

\subsection{Abundances of sn stars vs. CP stars}

We have determined the abundances of nine stars (including 6 sn stars, 1 sn candidate, and 2 non-sn stars) using synthetic spectra. To compare these values with the chemical abundances of CP stars, we present plots of abundance vs. atomic number in Figs. 4 and 5. The plots present abundance values relative to the Sun, i.e. values higher than zero are overabundant, while lower than zero are deficient. The $\mathrm{CP}$ stars present a range of possible abundance values rather than a single value for each chemical species. Their abundance pattern is presented as a "region" clearly demarcated by three continuous lines in Figs. 4 and 5. For the $\mathrm{HgMn}$ pattern we used $15 \mathrm{HgMn}$ stars listed in Adelman et al. (2006), with the addition of the HgMn star HD 165640 (Castelli \& Hubrig 2004). For the He-weak range we used four
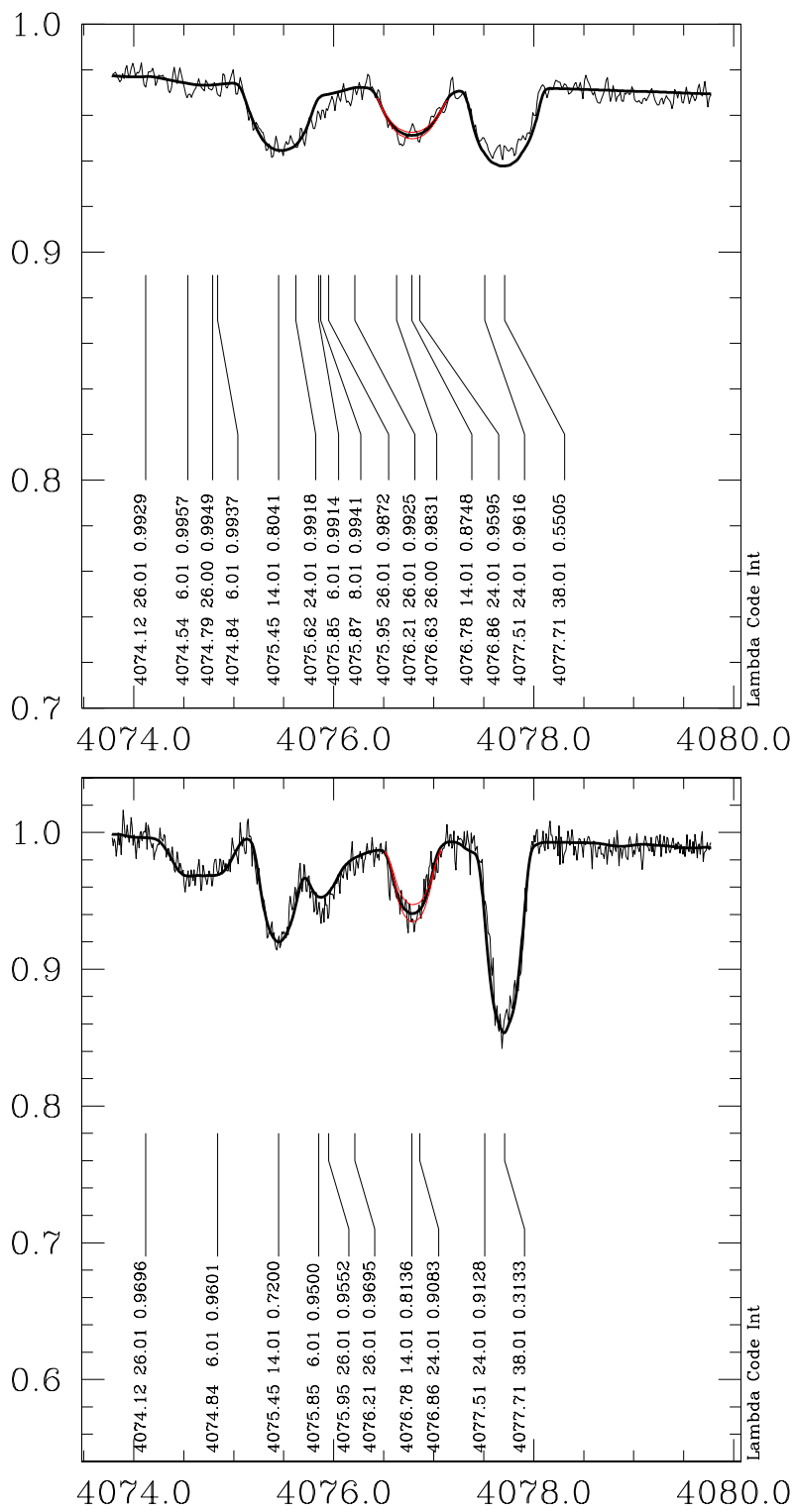

Fig. 3. Comparison of the spectral region near the line Si II 4076 for HD 162586 (upper panel) and HD 5737 (lower panel). The observed and synthetic spectra are shown by thin and thick lines, respectively. The 2 thin red curves correspond to $\pm 1 \sigma$ abundance variation of the synthethic spectra.

stars with abundances taken from the literature (Alonso et al. 2003), while for the $\lambda$ Boo pattern we used 12 stars taken from Heiter (2002). For the ApSi pattern, we used five stars taken from literature (López-García et al. 2001; López-García \& Adelman 1999, 1994; Albacete-Colombo et al. 2002). On the other hand, Figs. 4-5 show the abundance values derived in this work for our sample stars and the standard deviation of these values.

Next, some comments follow about individual stars, based mainly on the abundance values derived.

HD 5737: most of their abundance values agree with the pattern of a He-weak star (see Fig. 4), with He I clearly underabundant ( $\sim 0.7$ dex below solar), and with intense Ti II, Cr II, Fe II, and Sr II. We also identified $\mathrm{Cl}$ II in this star and determined the abundance using eight different lines. Lines of $\mathrm{Cl}$ II that are clearly identified are $4233.9 \AA$ and the doublet $5217.74 \AA$, $5217.94 \AA$ A. We derived for $\mathrm{Cl}$ II an overabundance higher than $\sim 1$ dex with a dispersion of 0.22 dex. In Fig. 6 we present two 

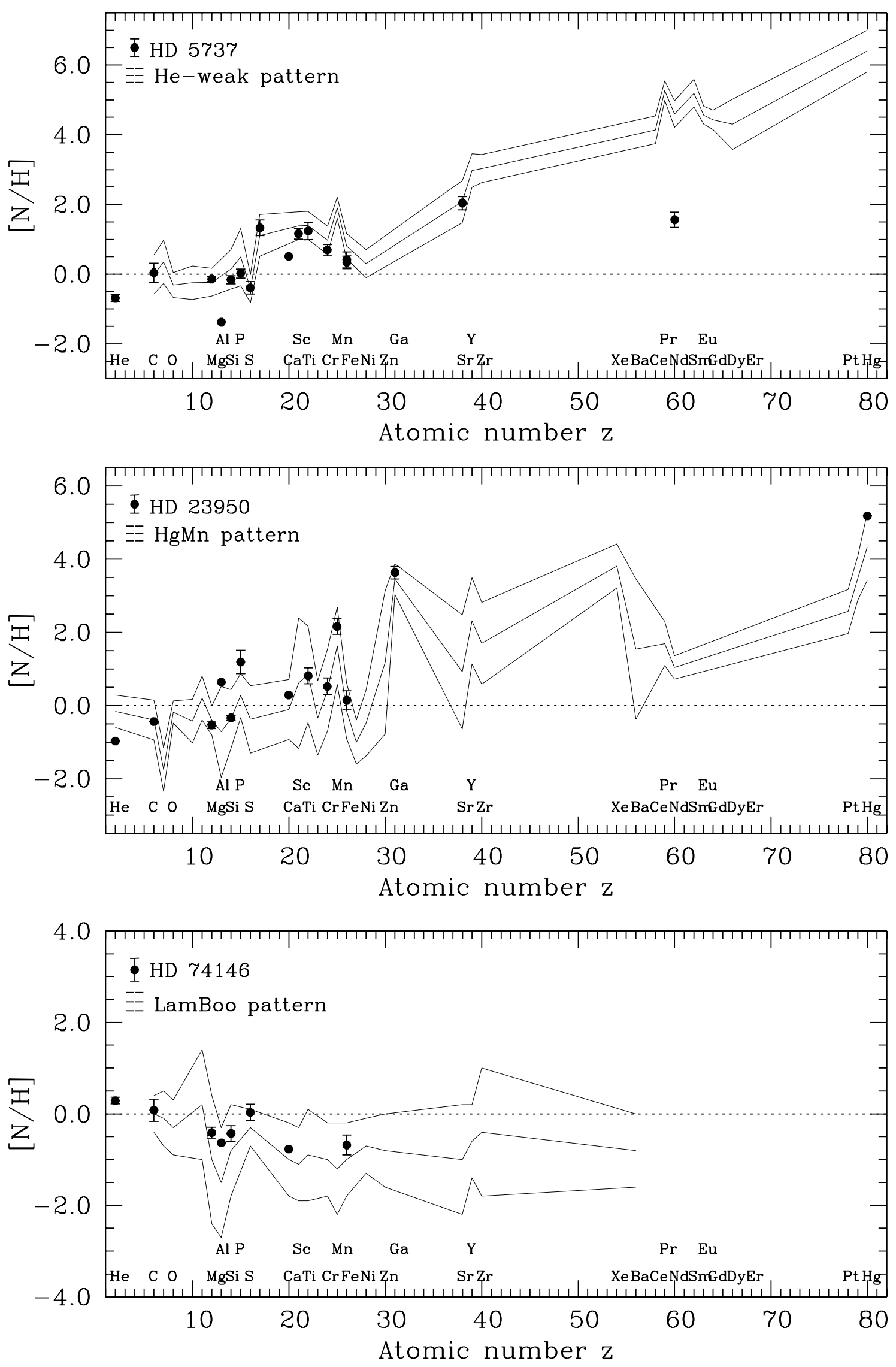

Fig. 4. Comparison of abundance patterns as function of the atomic number. Upper panel: HD 5737 (filled circles) vs. He-weak pattern (the 3 continuous lines). Middle panel: HD 23950 (filled circles) vs. HgMn pattern (the 3 continuous lines). Lower panel: HD 74146 (filled circles) vs. $\lambda$ Boo pattern (the 3 continuous lines). See text for more details. 

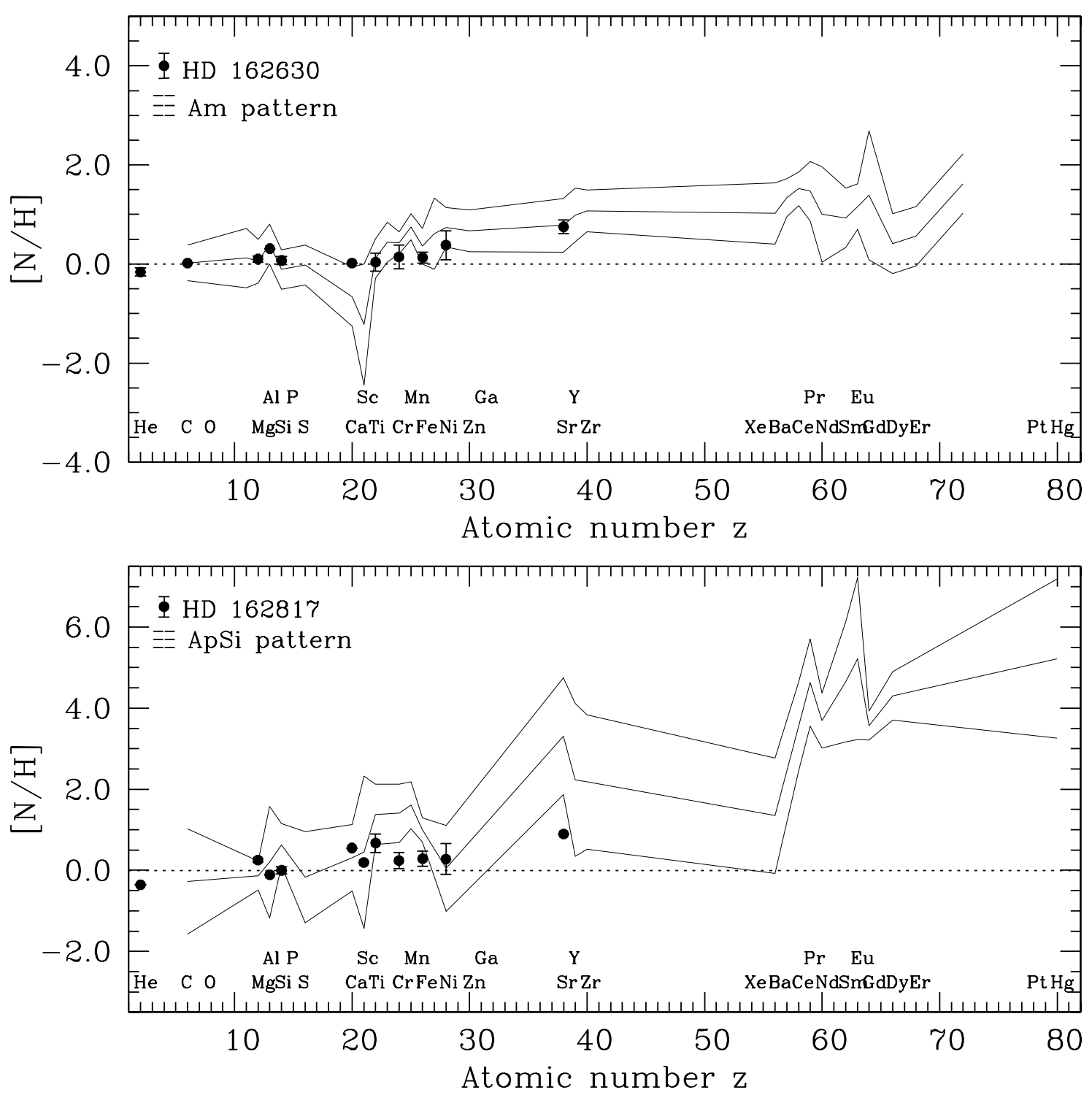

Fig. 5. Comparison of abundance patterns as function of the atomic number. Upper panel: HD 162630 (filled circles) vs. Am pattern (the 3 continuous lines). Lower panel: HD 162817 (filled circles) vs. ApSi pattern (the 3 continuous lines). See text for more details.

examples of the Cl II lines $3860.8 \AA$ and $4233.9 \AA$, together with the synthetic fits to the observed spectra.

HD 21071: this is slightly subsolar but otherwise normal star. For instance, the $\mathrm{He}$ and $\mathrm{Fe}$ abundances are -0.18 and -0.20 dex, respectively. This star shows no patterns like any of the classical CP stars.

HD 23950: this is clearly a HgMn star (see Fig. 4), with an excess of both Mn II and $\mathrm{Hg}$ II relative to the Sun (2.16 dex and 5.18 dex, respectively). In Fig. 7 we show the intense line Hg II 3984 identified in HD 23950, together with their synthetic spectra. The underabundances of C II ( -0.44 dex $), \mathrm{Mg}$ II ( $-0.53 \mathrm{dex})$, and Si II (-0.34 dex), together with overabundances of Ti II (0.81 dex), Cr II (0.52 dex), and Ga II (3.63 dex), follow the general trend of the HgMn pattern.

HD 74146: this object has underabundances of $\mathrm{Mg}$ II $(-0.41)$, Al II $(-0.63)$, Si II $(-0.43)$, Ca II $(-0.77)$, and Fe II $(-0.68)$ compared to the Sun. The subsolar value of Fe and other metals agree with a $\lambda$ Boo or a mild- $\lambda$ Boo star (see Fig. 4).

HD 162586: its abundances appear to be quite close to the solar values. This star shows no patterns like any of the CP stars.
HD 162630: this star presents solar or subsolar $\mathrm{He}$ $(-0.16$ dex relative to the Sun), with slight metallic overabundances of $\mathrm{Cr}$ II $(0.14 \mathrm{dex})$ and Fe II $(0.13 \mathrm{dex})$. The general pattern is similar to an Am or mild-Am star (see Fig. 5), including the overabundances of Al II (0.31 dex), Ni II (0.38 dex), and Sr II (0.75 dex).

HD 162678: this object present a slight deficiency of C II $(-0.38 \mathrm{dex})$ and Sc II (-0.29 dex $)$, however most elements show almost solar abundances (Mg II, Al II, Si II, Ca II, Cr II, and Fe II). This object does not follow the patterns of the classical CP stars, and present nearly solar abundances.

HD 162679: its abundances appear close to the solar values, except probably for Sc II (-0.45 dex). This object shows no patterns like any of the CP stars.

HD 162817: this object shows a subsolar He abundance $(-0.36$ dex relative to solar); however, its temperature $(10428 \mathrm{~K})$ is lower than the usual for He-weak stars with B spectral types (13000-15000 K). The common underabundaces of $\mathrm{Ca}$ and/or $\mathrm{Sc}$ expected in Am stars, or the intense lines of $\mathrm{Hg}$ and/or $\mathrm{Mn}$ in HgMn stars, are not observed in this object. HD 162817 presents overabundances of Ca II (0.55 dex relative to solar), 

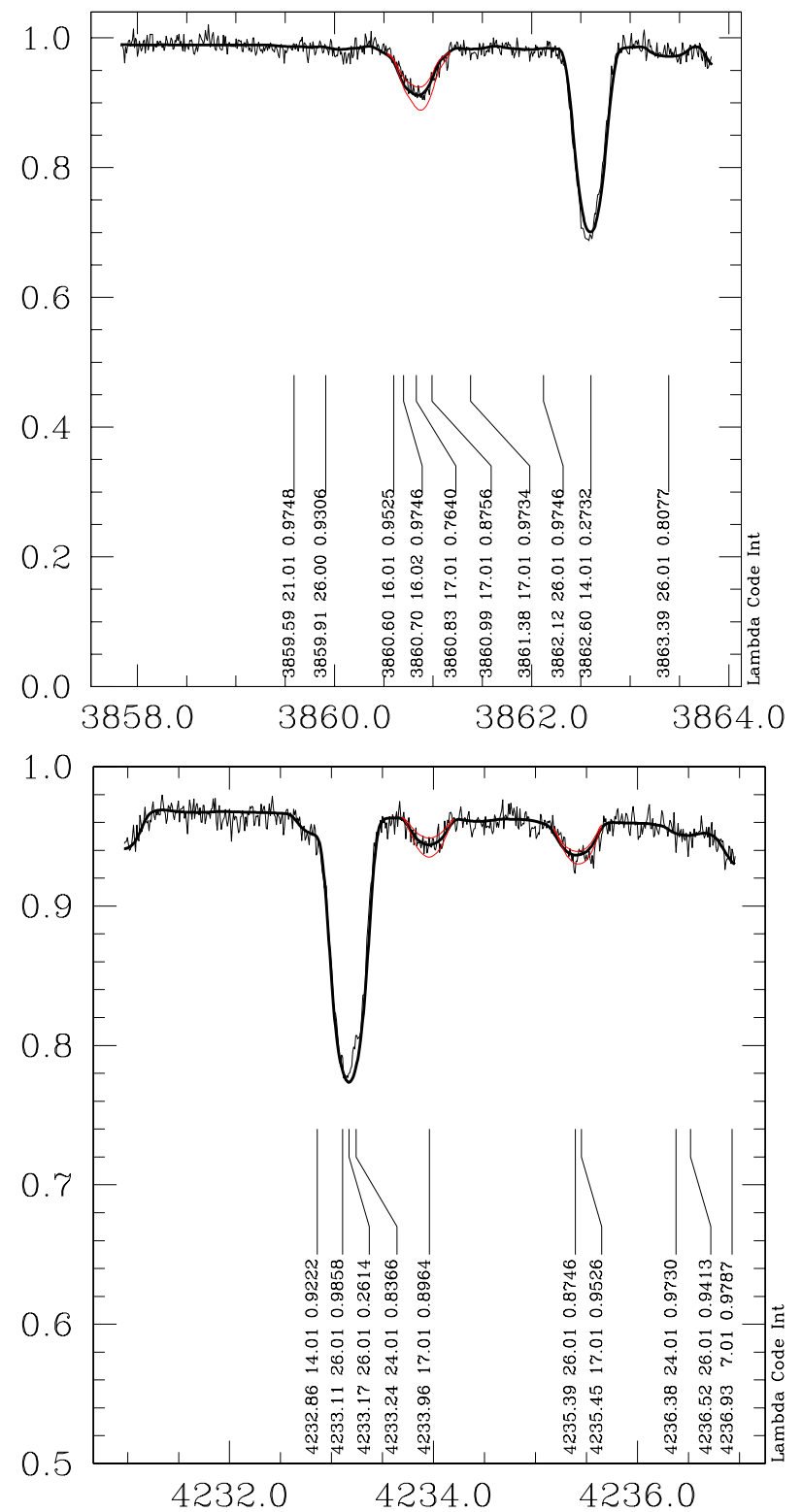

Fig. 6. Spectral region near the Cl II lines 3860 and 4233 (upper and lower panels, respectively) identified in HD 5737. The observed and synthetic spectra are shown by thin and thick lines, respectively. The 2 thin red curves correspond to $\pm 1 \sigma$ abundance variation of the synthethic spectra.

Sc II (0.19 dex), Ti II (0.67 dex), Cr II (0.24 dex), and Fe II (0.29 dex), similar to an Ap or mild-Ap (see Fig. 5) star.

Our sample includes He-weak stars (HD 5737), Am or mildAm stars (HD 162630), mild-Ap stars (HD 162817), HgMn stars (HD 23950), mild- $\lambda$ Boo stars (HD 74146), and stars with abundances close to solar (HD 21071, HD 162586, HD 162678, and HD 162679). Within a temperature range of $10300-14500 \mathrm{~K}$, $\sim 40 \%$ of the sn stars resulted CP stars. This value should be taken with caution due to how few stars are studied here. However, the sn stars display a variety of chemical peculiarities. No clear common spectral feature or abundance pattern is apparent, beyond the sn characteristics itself. From the point of view of the chemical composition of the atmospheres, the sn stars are a rather inhomogeneous group of stars. The sn characteristics appear in stars with very different chemical properties.

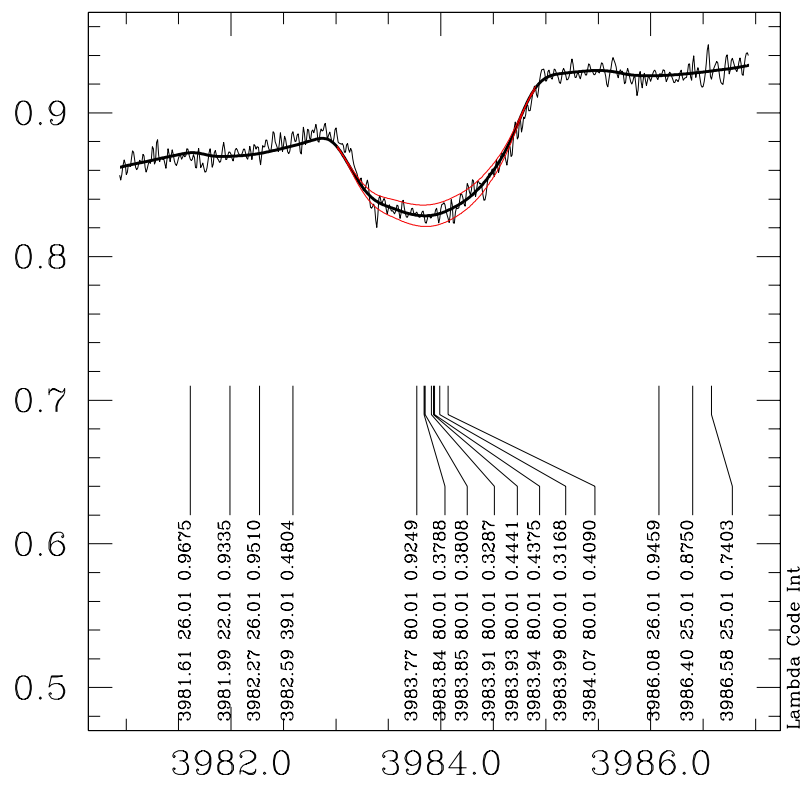

Fig. 7. Spectral region near the Hg II line 3984 identified in HD 23950. The observed and synthetic spectra are shown by thin and thick lines, respectively. The 2 thin red curves correspond to $\pm 1 \sigma$ abundance variation of the synthethic spectra.

We studied a sample of sn stars and not all of them display $\mathrm{CP}$ abundances, i.e. some sn stars present $\sim$ solar abundance values. There is a lack of a clear relationship between sn stars and $\mathrm{CP}$ stars. The anomalous chemical pattern of the CP stars is usually explained by the diffusion processes. A supposed relation sn-CP would indicate that the sn characteristics are present in those atmospheres with the physical conditions required by the diffusion to work efficiently. This is also supported by their low rotational velocities. However, the observational evidence shows that this does not seem to be the case. We show that the sn characteristics can coexist simultaneously with the abnormal abundances, but the chemical peculiarity is not a requirement for the sn phenomenon to appear.

Some stars in our sample agree with the general abundance pattern of the CP stars. However, they also show some discrepancies compared to the general pattern. A detailed inspection of classical CP stars shows that it is not unusual to find some starto-star differences in the abundances for each element. Most of the chemical anomalies observed in the CP stars are usually explained by the radiative diffusion (e.g. Michaud 1970; Michaud et al. 1976), which is expected to depend mainly on the effective temperature. However, the abundances could be significatively affected, for instance, by the presence of magnetic fields (see e.g. Michaud et al. 1981). There are other physical processes that could work in addition to the diffusion modifying the abundances, such as mass loss (e.g. Vick et al. 2008, 2010) or turbulence mixing (e.g. Richer et al. 2000; Richard et al. 2001). However, it is difficult to determine which of these mechanisms could produce the resulting abundances. For instance, the abundances of Sirius A and of the Hyades star 68 Tau could be explained equally well by either mass loss or turbulence in addition to diffusion (Richer et al. 2000).

Even for the case of the superficially "normal" stars, the elemental abundances of late-B to F stars show a range of possible abundances (Adelman \& Unsuree 2007; Hempel \& Holweger 2003). Adelman \& Unsuree (2007) found that the abundance distribution in normal A stars overlap with those of the Am stars. Most normal A stars are likely to have a few values that are of order \pm 0.40 dex from solar, and this indicates that a common 
phenomenon could produce the anomalies, such as a combination of diffusion and mass loss. It is not uncommon to find stars considered "normal" with no exactly solar abundance values. In our sample of sn stars, three objects display abundances within this approximate range (HD 21071, HD 162586, and HD 162678). Hempel \& Holweger (2003) studied 27 B stars classified as normal and detected anomalous rather than solar abundances in different elements $(\mathrm{O}, \mathrm{Mg}, \mathrm{Ca}, \mathrm{Fe}, \mathrm{Sr}, \mathrm{Ba})$. They show that the anomalies do not occur to the same extent in all stars but instead show star-to-star variations. The authors suggest that a counterpart to diffusion such as meridional mixing is operating in the atmospheres of these normal stars.

\subsection{Other properties of sn stars}

We found no clear relation between the fundamental parameters ( $T_{\text {eff }}, \log g$ and age) and the photospheric chemical composition of the sn stars. This is probably related to a low statistical significance (only nine stars). On the other hand, our sample includes five stars that belong to the same cluster (NGC 6475). These five stars (HD 162586, HD 162630, HD 162678, HD 162679, and HD 162817) allow a comparison where the age and the initial composition effects are negligible. The group include two non-sn stars (HD 162630 and HD 162817) and the other three are sn stars. The range of temperature of the three sn stars is between $10219 \mathrm{~K}-12500 \mathrm{~K}$ i.e. mixed with the temperatures of the non-sn stars (10428 K and $10625 \mathrm{~K})$. The temperature alone does not seem to determine the sn characteristics.

The rotational velocities of the three sn stars in NGC 6475 $\left(27 \mathrm{~km} \mathrm{~s}^{-1}, 40 \mathrm{~km} \mathrm{~s}^{-1}\right.$, and $\left.40 \mathrm{~km} \mathrm{~s}^{-1}\right)$ are lower than the $v \sin i$ values of the two non-sn stars of the same cluster $\left(49 \mathrm{~km} \mathrm{~s}^{-1}\right.$ and $75 \mathrm{~km} \mathrm{~s}^{-1}$ ). The apparent preference of the sn stars for objects with low $v \sin i$ agrees with the result of Mermilliod (1983). However, this should be taken with caution owing to the low number of objects studied here.

Moujtahid et al. (1998) studied the spectrophotometric variations of $49 \mathrm{Be}$ and shell-like stars. They found six with relatively low $v \sin i$ values that are candidates to exhibiting "spectrophotometric shell behaviour". These spectrophotometric criteria should be taken with caution because the presence of absorption lines is required for a shell star. However, none of the six stars proposed by Moujtahid et al. (1998) present evidence of shell absorption lines (Rivinius et al. 2006). On the other hand, as mentioned in the Introduction, Neiner et al. (2005) identified a group of $\mathrm{Be}$ and shell stars in the field of view of the CoRoT satellite. In particular, one of the stars they discovered (HD 174512) is classified either as a slowly rotating $\left(v \sin i \sim 20 \mathrm{~km} \mathrm{~s}^{-1}\right.$ ) shell star or as a Herbig Be star. If the shelllike nature of this object is confirmed, this could be the first star of this class with a low projected rotational velocity.

For the case of $\mathrm{CP}$ stars, it is not fully understood how the abundances are affected by stellar rotation. Once the He convection zone has disappeared in stars with $v \sin i$ lower than $75 \mathrm{~km} \mathrm{~s}^{-1}$ (Charbonneau \& Michaud 1988), stable atmospheres and diffusion are expected to occur. Charbonneau \& Michaud (1991) carried out detailed calculations of diffusion in the presence of meridional circulation and turbulence. They conclude that no dependence of abundance anomalies on $v \sin i$ is expected for most elements, because diffusion time scales are much shorter than a meridional circulation time scale. However, the authors note that their calculations do not imply that there should be no correlation at all between rotation and abundance anomalies. Some effects, such as the dependence of the He cutoff velocity on $\log g$ and on stellar evolution, could lead to a weak anticorrelation between abundances and rotation. Burkhart (1979) did not find any decrease in abundance anomalies with increasing $v \sin i$. On the other hand, Boyarchuk \& Savanov (1986) show that the Vanadium overabundance decrease with increasing rotational rate. The lack of correlation between abundances and rotation show that the meridional circulation has little influence on chemical separation for $v \sin i<100 \mathrm{~km} \mathrm{~s}^{-1}$ (Charbonneau \& Michaud 1991).

\subsection{Line profiles of sn stars}

Abt \& Levato (1977) mentioned that their 14 sn stars have both sharp and broad lines of He I, along with sharp metallic lines. Another example is presented in Fig. 2 of Abt (1978), where the sn star HD 36392 presents both broad lines of He I (4009 $\AA$, $4026 \AA, 4144 \AA$ ) and sharp "normal" lines of He I (4121 $)$. In other words, sn stars usually present a sharp lined spectra, except for some particular He I lines (4009 ̊, $4026 \AA$, $4144 \AA$ ). However, in our sample the line $4009 \AA$ is weakly present or not seen in most of the stars, while the line $4026 \AA$ is always present, since it is probably the clearest example of a broad He I line in the sn stars of our sample. It is notable that we have identified the sn characteristic in our group with only one broad He I line in common. This may give rise to the question about whether the sn stars are unusual in their He lines compared to other normal stars. This topic is addressed in this section.

We explore three different physical proccesses, in order to determine whether they have some contribution (or not) to the particular line profiles observed in the sn stars. The processes discussed include NLTE effects, broadening mechanisms of spectral lines, and the possible stratification of the atmospheres. In particular, we focus on the broad $\mathrm{He}$ I lines that are present in the sn spectra.

\subsubsection{NLTE effects in the He I lines}

Different lines of He I are more or less sensitive to the NLTE effects. To verify possible presence NLTE effects in the He I lines, we compared in Fig. 8 the observed vs. LTE synthetic spectra for the line $4921 \AA$, which is one of the most sensitive He I lines to the NLTE effects (e.g. Przybilla et al. 2011). The example corresponds to the sn stars HD 162586, HD 162679, HD 21071, and HD 74146, where this line is intense, and then the NLTE effects should be noted first. The agreement between observed and LTE synthethic spectra for this line shows that NLTE effects are not clearly detected in the He I profiles of these stars.

If NLTE effects are present in the profiles of He I lines, they are mainly noted as an enhancement in the cores of lines (e.g. Przybilla et al. 2011). The cores of the lines are formed in the higher layers of the atmosphere where the density is lower, the number of interactions between particles decrease, and the radiation field tends to dominate. However, we have observed a broadening in the wings of He I lines such as $4026 \AA$ in our sample rather than an enhancement in the cores. In other words, NLTE effects and the sn characteristics involve, in principle, different parts of the He I profiles (cores and wings, respectively). Also, NLTE effects are first noted in their most sensitive lines, such as $4921 \AA, 5875 \AA$, and $6678 \AA$ (e.g. Przybilla et al. 2011), while the sn characteristics are identified first in other lines such as $4026 \AA$ in our sample. Although NLTE effects could not be completely ruled out, it does not seem that the sn characteristics are directly related to the NLTE effects. 

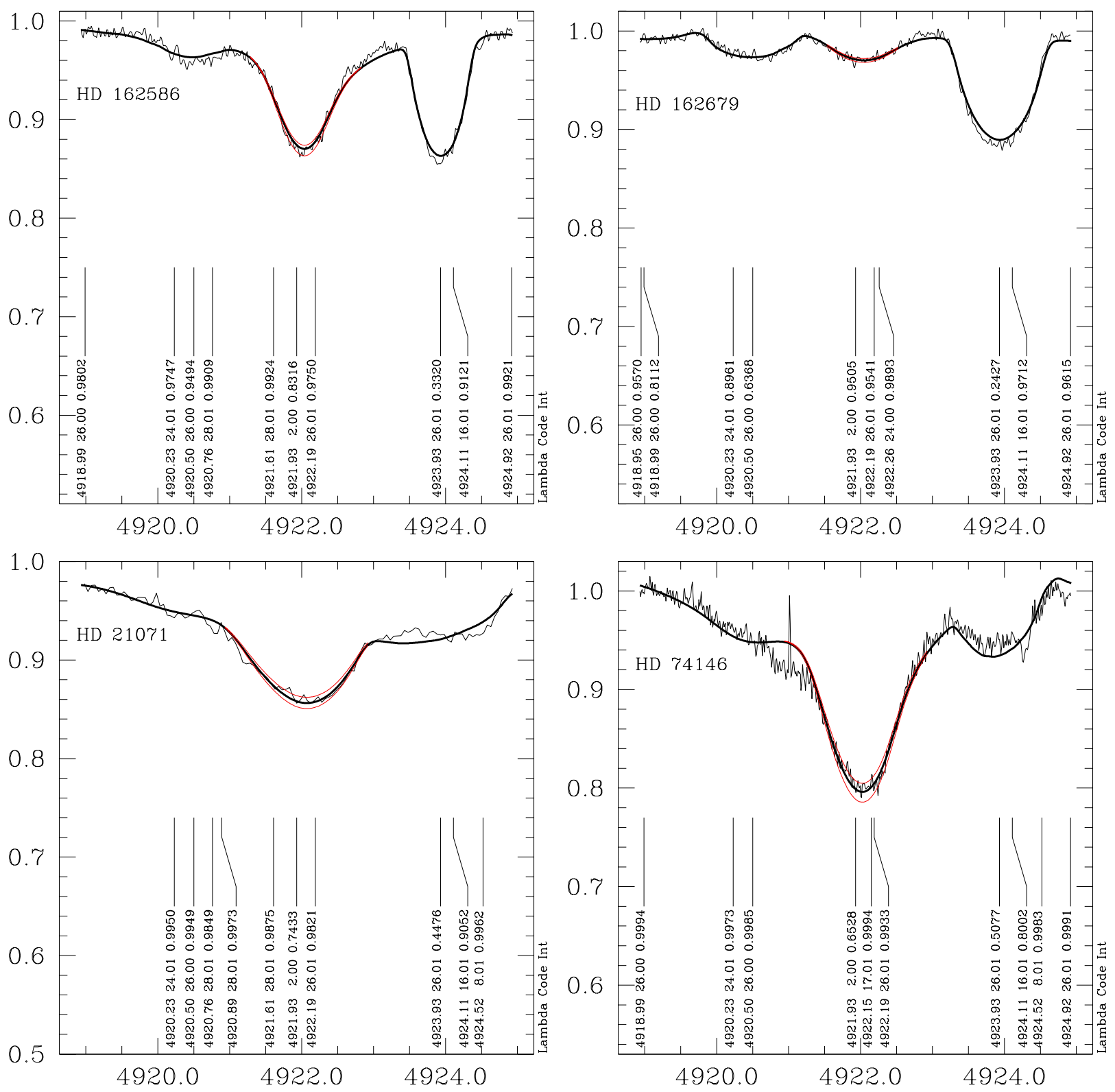

Fig. 8. Spectral region near the He I line $4921 \AA$ A. The observed and synthetic spectra are shown by thin and thick lines, respectively. The panels correspond to the stars HD 162586, HD 162679, HD 21071, and HD 74146. The 2 thin red curves correspond to $\pm 1 \sigma$ abundance variation of the synthethic spectra.

\subsubsection{Broadening of He I lines}

The intrinsic line profiles in the synthetic spectra take the effects of natural, Stark, Van der Waals, and thermal Doppler broadening into account. The computed spectra must be convolved with the instrumental profile and broadened by the star rotation. For temperatures higher than around $10000 \mathrm{~K}$, the $\mathrm{H}$ is mainly ionized, and the dominant line broadening mechanism in B stars is the quadratic Stark effect, owing to interactions of absorbing atoms with charged particles (e.g. Gray 2005). There are different methods of computing the Stark broadening $\Gamma$ function of the spectral lines (e.g. Cowley 1971; Kurucz et al. 1974; Popovic et al. 2001). Kurucz et al. (1974) and Cowley (1971) describe two separate methods of calculating the Stark broadening $\Gamma$ independent of temperature, while the Popovic et al. (2001) method scales the Kurucz et al. (1974) broadening by the square root of the temperature. For most of the spectral lines, the ATLAS9 and SYNTHE codes use the Kurucz et al. (1974) method for calculating the Stark broadening.
The He I lines could be incorrectly modelled if the Stark broadening is not adequately taken into account (e.g. Przybilla et al. 2011). To improve the modelling of the He I profiles, we used detailed line broadening tables for the line $4471 \AA$ (Barnard et al. 1974) and for the lines $4026 \AA, 4387 \AA$, and $4922 \AA$ (Shamey 1969). Most of these profiles have been computed assuming quasistatic broadening by ions and impact broadening by electrons, with the semiclassical broadening theory for overlapping lines of Griem et al. (1962). Although these detailed calculations do not include all lines of He I, they do include the line $4026 \AA$, which clearly displays the sn characteristics (a broad He I line) in all stars of our sample.

The Figs. 9 and 10 show the observed vs. synthethic spectra derived for the line $4026 \AA$, including the detailed Stark broadening tables in the calculation. We mentioned that the observed broadening in this line is the clearest example of the sn characteristics (broad He I line) in our sample. We note small differences between observed and synthethic spectra for the stars 
C. Saffe and H. Levato: On the nature of sn stars. I.
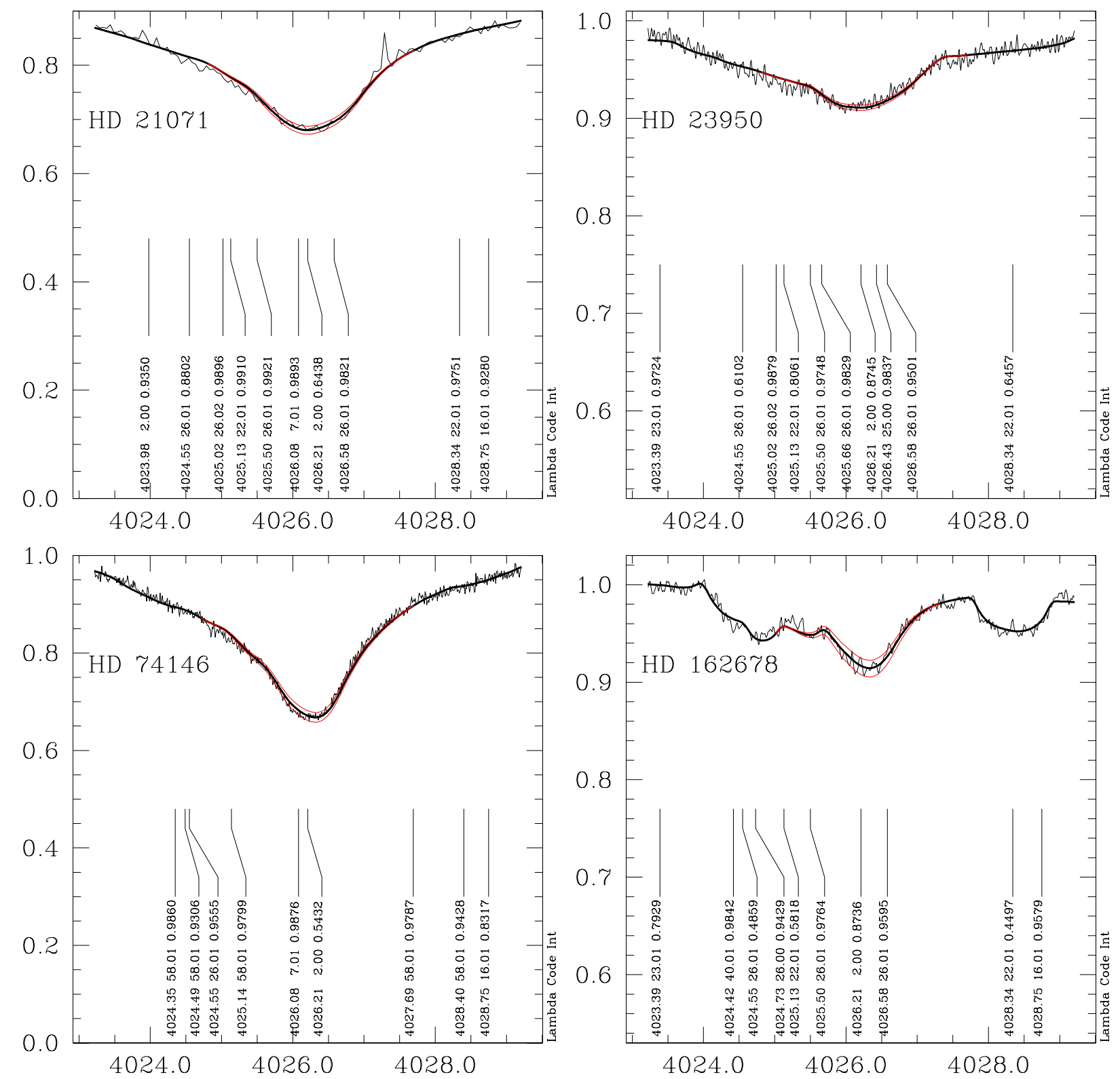

Fig. 9. Spectral region near the He I line $4026 \AA$ for a sample of sn stars. The observed and synthetic spectra are shown by thin and thick lines, respectively. The panels correspond to the stars HD 21071, HD 23950, HD 74146 and HD 162678. The 2 thin red curves correspond to $\pm 1 \sigma$ abundance variation of the synthethic spectra.

HD 5737, HD 162586, and HD 162679 (see Fig. 10), although (even for these stars) the general broadening seems to agree with observations. The small differences in these three stars do not seem to be NLTE effects, because this is not detected in the more sensitive He I line $4921 \AA$ (see next section for another possible effects). We succesfully fitted the He I $4026 \AA$ in most sn stars (4 out of 7) by adopting the correct Stark broadening tables, including the instrumental and rotational convolutions. This modelling seems to be enough to describe the profile of the line $4026 \AA$, without needing to include an extra broadening mechanism, except probably for HD 5737, HD 162586, and HD 162679.

There is good agreement between the He I abundance values derived from the broad lines (4009 $\AA, 4026 \AA, 4144 \AA$ ) and from the rest of the He I lines. There is no clear tendency between them in the sn stars, i.e. the abundances obtained from the broad He I lines are not greater or lower than the sharp He I lines.

\subsubsection{Search for stratification of He I lines}

Studying He-peculiar stars in particular, Vauclair et al. (1991) predict that the He abundance should decrease with depth in the atmosphere of He-rich stars and increase in He-weak stars; i.e. both should present He stratified atmospheres. Farthmann et al. (1994) proposed that the excesively broad wings of the He I line $4471 \AA$ observed in the He-weak star HD 49333 are due to a vertical He stratification. They require the use of stratified instead of homogeneous models to account for the observed wings in this line, by adopting a greater He abundance in the deepest layers. Leone \& Lanzafame (1997) confirm the Vauclair et al. result for He-rich stars; however, they found the contrary effect for Heweak stars (i.e. the abundance decrease with depth) by studying the He I red spectral transitions $6678 \AA, 7065 \AA$, and $7281 \AA$. This is also in contrast to Farthmann et al. (1994), in which the abundance increases with depth in the atmosphere. 

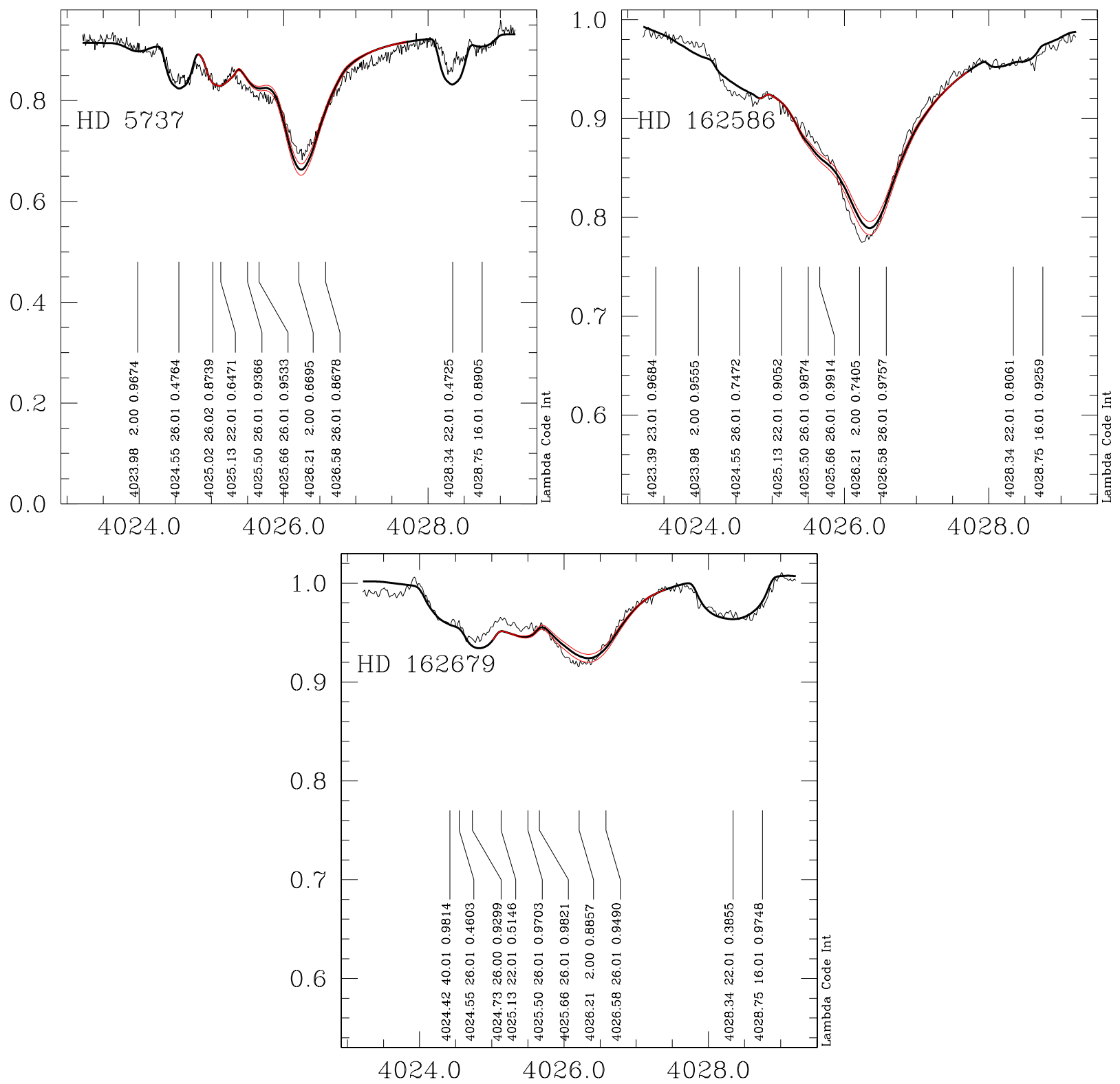

Fig. 10. Spectral region near the He I line $4026 \AA$ for a sample of sn stars. The observed and synthetic spectra are shown by thin and thick lines, respectively. The panels correspond to the stars HD 5737, HD 162586 and HD 162679 for which the synthethic spectra does not perfectly fit the observations. The 2 thin red curves correspond to $\pm 1 \sigma$ abundance variation of the synthethic spectra.

More recently, Zboril (2005) has found that 5 out of $19 \mathrm{He}-$ rich stars are probably He-stratified, studying the $4026 \AA$ line profiles. Catanzaro (2008) finds that the He I abundance decrease towards the deepest layers in the He-rich star HD 145792 by using a plot of He I abundance vs. optical depth for different lines. He also noted that the impossibility of fitting the line $4026 \AA$ with a single abundance value is a sign of stratification. For this line, the author reproduce the core but not the red wing of the profile. Although there are some discrepancies if the He increase or decrease with depth in He-peculiar stars, the mentioned works agree that the $\mathrm{He}$ is possibly stratified and that this could affect the profiles of $4471 \AA$ (Farthmann et al. 1994) and 4026 A (Zboril 2005; Catanzaro 2008).

Leone (1998) found that the He-rich star HD 37017 is He stratified in their magnetic pole regions. This variable star show a stratified atmosphere (in a plot of abundance vs. depth) when observed in the phase of helium maximum strength, however when looking in the minimum helium phase, the star appears to be unstratified. In other words, an analysis of a single observation does not seem to completely rule out the possibility of stratification.

In Fig. 11 we present a plot of abundance vs. optical depth for the He I lines measured in the sn stars, including the error bars of the points. The significance of the linear fit to the data could be estimated by comparing the slope $m$ of the fits with their corresponding error $\sigma_{m}$. The y values were displaced vertically in the figure to avoid the superposition of points. The cores and wings of the lines are formed mainly in the higher and lower layers of the atmosphere, respectively. Then, a possible wing broadening in the He I lines would correspond to an increase in the He abundance with depth (e.g. Farthmann et al. 1994). From Fig. 11, this effect seems be present in the sn stars HD 5737 $\left(m \sim 3.4 \sigma_{m}\right)$, HD $162678\left(m \sim 2.6 \sigma_{m}\right)$, and HD 162679 $\left(m \sim 4.6 \sigma_{m}\right)$, and less clearly in HD $162586\left(m \sim 1.5 \sigma_{m}\right)$.

We caution that the low number of points presented in Fig. 11, together with the error bars of the individual 

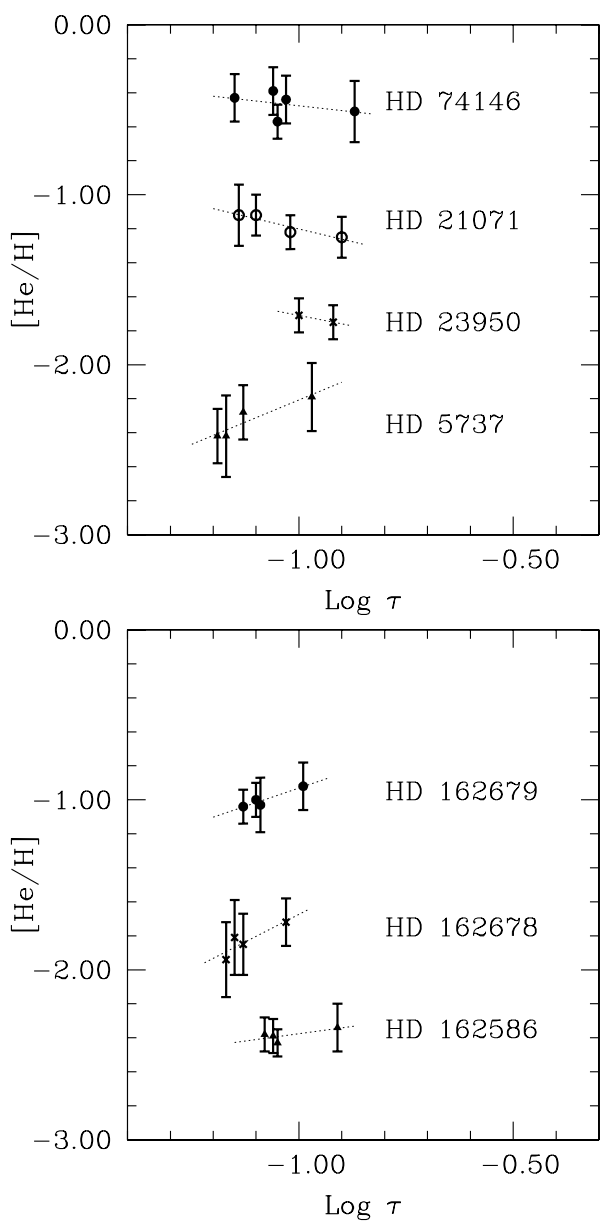

Fig. 11. Abundances of He I lines vs. optical depth for the sample of sn stars. The $y$-values were displaced vertically to avoid the superposition of points. The dotted line shows a linear fit to the data.

measurements, could diminish the significance of a possible trend. However, these plots give a first indication of a possible stratification in these atmospheres (e.g. Catanzaro 2008). We showed that HD 5737 is a He-weak star, so then a possible Hestratification could not be discarded. The stars HD 162678 and HD 162679 present almost solar He abundance values, within $\sim 0.13$ dex. From Fig. 11 a possible increase in the He abundance with depth could not be ruled out in the stars HD 5737, HD 162679, and less probably in HD 162586. For these stars, a stratified model atmosphere probably improves the agreement with the observed profile of the He I line $4026 \AA$.

Figure 11 suggests that different abundance values could correspond to different depths in the atmosphere. If the abundance increases with depth, the cores of the individual lines should be adjusted with a lower abundance value than for the wings. However, it is difficult to see this effect in the profile of a single line such as He I $4026 \AA$ (see Fig. 10). We note that the final abundance value adopted for these lines corresponds to the best fit including both the core and the wings. The curves of $\pm 1 \sigma$ abundance values are indicative of the effect of increase or decrease in the abundance of the line. A variation in the abundance is first noted in the core of the profile, and then it is (progresively) less intense towards the wings. For the stars HD 162586 and HD 162679, the blue wing of He I $4026 \AA$ is not properly fitted (see Fig. 10). Many attempts were made, although without success. This is probably due to strong blends with Fe II $4024.55 \AA$, Fe III $4025.02 \AA$, and Ti II $4025.13 \AA$ for the case of HD 162679. The red wings are better fitted although not perfectly. The cores of He I $4026 \AA$ seem to be slightly blueshifted in wavelength only in these two sn stars, and cannot be properly fitted even for different abundance values. For the star HD 5737, we note that the red wing of He I $4026 \AA$ (roughly between $\sim 4026.2$ and $\sim 4028 \AA$ ) could not be fitted by varying the abundance. The core of the line shows better agreement with a lower abundance (which corresponds to an increase in abundance with depth), although it does not fit perfectly. For the three stars of Fig. 10, it is very difficult to fit the line He I $4026 \AA$, even when adopting different abundance values for the cores and wings. This suggests that another effect is probably superimposed, such as a non-uniform distribution of He in the surface (e.g. Bohlender 1989).

The He-strong sn star $\delta$ Ori $\mathrm{C}$ was studied by Bohlender (1989) using a high-resolution spectra taken at the CFHT. The author found that the He I line profiles cannot be reproduced with a uniform atmospheric He abundance. He proposed two explanations: a non-uniform distribution of $\mathrm{He}$ in the stellar surface or a vertical He stratified atmosphere. This work shows that the He-stratified atmospheres cannot be ruled out in at least some sn stars by using the plots of abundance vs. depth (see Fig. 11). The non-uniform distribution of He could be analysed by taking spectra at different rotational phases. The study presented here is mostly based on a single observation of the sn stars. A further study of variability in the He I lines would help determine if a possible non-uniform He distribution could play a role in the sn stars.

In the previous sections, we used a model atmosphere with given physical conditions and appropriate line broadening, and fitted both broad and sharp lines of four out of seven sn stars. In particular, we properly calculated the broad He I line profiles that characterize and help define the class of sn stars. Therefore we showed that at least in some stars the broadening could have originated in the atmospheres of these stars. There is no need to suppose another mechanism or an extra-broadening source for the He lines. The usual radiative transfer processes that give rise to the "normal" He line formation and include the Stark broadening seem to be enough to describe the line spectra of sn stars. For the three other stars, the small differences in the profiles seem to be due, at least in part, to stratification (increase He abundance with depth) in these atmospheres, although a non-uniform He surface distribution could not be ruled out. We suggest more observations and the use of stratified model atmospheres to properly describe the He I profiles in these sn stars.

\section{Concluding remarks}

We have derived the chemical abundances of nine stars, including seven sn stars and two non-sn stars that belong to four open clusters, for 23 different chemical species. We used the calibration of Napiwotzki et al. (1993) with the Strömgren uvby $\beta$ photometry of Hauck \& Mermilliod (1998) to derive the fundamental parameters $T_{\text {eff }}$ and $\log g$. These parameters were corrected for the He-weak star HD 5737 and for the HgMn star HD 23950, based on the abnormal colour present in CP stars. We improved them by requiring ionization and excitation equilibrium of Fe lines. We derived the abundances by fitting synthetic and observed spectra using the program SYNTHE, together with ATLAS9 model atmospheres. The complete line-by-line 
abundances, including plots of the synthetic spectra, are available on the $w_{e b}{ }^{7}$.

We derived the rotational velocities homogeneously for our sample stars, and we agreed with the conclusion of Mermilliod (1983) that the sn stars present relatively low projected rotational velocities. Although shell-like stars usually present higher rotational velocities, the shell-like nature cannot be completely ruled out based solely on the rotational properties. We also compared five stars that belong to the same cluster (NGC 6475), and show that the sn characteristics appear in three stars with lower rotational velocity. This apparent preference for stars with lower $v \sin i$ values should be taken with caution owing to how few objects are studied here.

We derived the abundances for the stars in our sample and compared them with the abundance values of CP stars. We verified that approximately $\sim 40 \%$ of the sn stars studied are CP stars, within a temperature range of $10300 \mathrm{~K}-14500 \mathrm{~K}$; however, no clear common spectral feature or abundance value is apparent for the sn stars. As a group they present a rather inhomogeneous chemical composition. We also showed that not all sn stars display classical $\mathrm{CP}$ abundances; i.e. some sn stars present a $\sim$ solar abundance pattern. There is lack of a clear relationship between sn stars and CP stars. Although the sn characteristics can coexist simultaneously with the abnormal abundances, the chemical peculiarity does not seem to be a requirement for the appearance of the sn phenomena. We find no clear relation between the fundamental parameters ( $T_{\text {eff }}, \log g$, and age) and the chemical composition of the sn stars. The small number of stars analysed could prevent us to detect some clear trend.

We studied the possible contribution of three different processes to the broad He I lines present in the sn stars. We used a model atmosphere trying to account for both sharp and broad lines observed in the sn stars. Although NLTE effects could not be completely ruled out in the He I lines, it seems that they are not directly related to the broad He I profiles observed in the sn stars. The broad line He I $4026 \AA$ is the clearest example of the sn characteristics in our sample. By using the appropriate Stark broadening tables, we succesfully fit this line in four out of seven sn stars, while small differences appear in the other three stars. We conclude that the broad He I lines that characterize the sn class could be modelled by the usual radiative transfer process with Stark broadening, at least in some of the sn stars. In other words, the observed broadening seems to be related to the "normal" He line formation that originated in the atmospheres of these stars. Studying the plots of abundance vs. depth for the He I lines showed that some sn stars are also probably stratified in He. However, a further study of variability in the He I lines would help to determine if a possible non-uniform He distribution could also play a role in these sn stars.

Acknowledgements. The authors thank Drs. R. Kurucz, F. Castelli, P. Bonifacio and L. Sbordone for making their codes available to them. We appreciate support from CONICET through grant PIP 1113-2009. We also thank the anonymous referee whose suggestions improved the paper.

\section{References}

Abt, H. 1978, PASP, 90, 692

Abt, H. 1979, AJ, 230, 485

Abt, H. 2009, AJ, 138, 28

\footnotetext{
7 http://icate-conicet.gob.ar/saffe/sn/Html/Salida7. html
}

Abt, H., \& Levato, H. 1977, PASP, 89, 797

Abt, H., \& Moyd, K. I. 1973, ApJ, 182, 809

Abt, H., \& Morrel, N. 1995, ApJS, 99, 135

Adelman, S. J., \& Rayle, K. E. 2000, A\&A, 355, 308

Adelman, S. J., \& Unsuree, N. 2007, Baltic Astron., 16, 183

Adelman, S. J., \& Yuce, K. 2010, Astron. Nachr., 331, 785

Adelman, S. J., Adelman, A. S., \& Pintado, O. 2003, A\&A, 397, 267

Adelman, S., Caliskan, H., Gulliver, A. F., \& Teker, A. 2006, A\&A, 447, 685

Albacete-Colombo, J. F., López-García, Z., Levato, H., Malaroda, S. M., \& Grosso, M. 2002, A\&A, 392, 613

Alonso, M. S., López-García, Z., Malaroda, S., \& Leone, F. 2003, A\&A, 402, 331

Barker, P. K., Brown, D. N., Bolton, C. T., \& Landstreet, J. D. 1982, in Advances in Ultraviolet Astronomy. The Fourth Year of IUE, eds. Y. Kondo \& J. Mead, NASA CP-2238, 589

Barnard, A. J., Cooper J., \& Smith, E. W. 1974, JQSRT, 14, 1025

Barrado y Navascués, D., Stauffer, J. R., \& Patten, B. 1999, AJ, 522, L53

Bohlender, D. 1989, AJ, 346, 459

Boyarchuk, A., \& Savanov, I. 1986, in Upper Main Sequence Stars with Anomalous Abundances, eds. C. R. Cowley, M. M. Dworetsky, \& C. Megessier (Dordrecht: Reidel), 433

Brown, D. N., Shore, S. N., Barker, P. K., \& Sonneborn, G. 1984, in The future of the ultraviolet astronomy based on six years of IUE research, eds. J. Mead, R. D. Chapman, \& Y. Kondo, NASA CP-2349, 487

Brown, D. N., Shore, S. N., \& Sonneborn, G. 1985, AJ, 90, 1354

Burkhart, C. 1979, A\&A, 74, 38

Castelli, F., \& Hubrig, S. 2004, A\&A, 425, 263

Catanzaro, G. 2008, MNRAS, 385, L33

Charbonneau, P., \& Michaud, G. 1988, ApJ, 327, 809

Charbonneau, P., \& Michaud, G. 1991, ApJ, 370, 693

Cowley, C. R. 1971, The Observatory, 91, 139

Dekker, H., D’Odorico, S., Kaufer, A., Delabre, B., \& Kotzlowski, H. 2000, in Proc. SPIE 4008, eds. M. Iye, \& A. F. Moorwood, 534

Farthmann, M., Dreizler, S., Heber, U., \& Hunger, K. 1994, A\&A, 291, 919

Fuhr, J. R., \& Wiese, W. L. 2006, J. Phys. Chem. Ref. Data, 35, 4

Gratton, R. G., Carretta, E., Eriksson, K., \& Gustafsson, B. 1999, A\&A, 350, 955

Gray, D. F. 2005, Observation and Analysis of Stellar Photospheres (Cambridge University Press)

Grevesse, N., Noels, A., \& Sauval, A. 1996, Proc. the sixth annual October Astrophysics Conference in College Park, Maryland, eds. S. S. Holt, \& G. Sonneborn (San Francisco: ASP), ASP Conf. Ser., 99, 117

Griem, H. R., Baranger, M., Kolb, A., \& Oertel, G. 1962, Phys. Rev., 125, 177

Hauck, B., \& Mermilliod, M. 1998, A\&AS, 129, 431

Heiter, U. 2002, A\&A, 381, 959

Hempel, H., \& Holweger, H. 2003, A\&A 408, 1065

Kaufer, A., Stahl, O., Tubbesing, S., et al. 1999, The Messenger, 95, 8

Kurucz, R. L. 1993, ATLAS), Stellar Atmosphere Programs and $2 \mathrm{~km} \mathrm{~s}^{-1}$ grid, Kurucz CM-ROM No. 13 (Cambridge, MA: Smithsonian Astrophysical Observatory)

Kurucz, R. L., \& Avrett, E. H. 1981, SAO Special Report No. 391

Kurucz, R. L., Peytremann, E., \& Avrett, E. 1974, Blanketed model atmospheres for early-type stars (Washington: Smithsonian Institution)

Leone, F. 1998, CoSka, 27, 285

Leone, F., \& Lanzafame, A. C. 1997, A\&A, 320, 893

Leone, F., \& Manfré, M. 1997, A\&A, 320, 257

López-García, Z., \& Adelman, S. J. 1994, A\&AS, 107, 353

López-García, Z., \& Adelman, S. J. 1999, A\&A, 137, 227

López-García, Z., Adelman, S. J., \& Pintado O. I. 2001, A\&A, 367, 859

Mayor, M., Pepe, F., Queloz, D., et al. 2003, The Messenger, 114, 20

Mermilliod, J.-C. 1983, A\&A, 128, 362

Meynet, G., Mermilliod, J.-C., \& Maeder, A. 1993, A\&AS, 98, 477

Michaud, G. 1970, ApJ, 160, 641

Michaud, G. 1982, ApJ, 258, 349

Michaud, G., Charland, Y., Vauclair, S., \& Vauclair, G. 1976, ApJ, 210, 447

Michaud, G., Charland, Y., \& Megessier, C. 1981, A\&A, 103, 244

Moujtahid, A., Zorec, J., Hubert, A. M., Garcia, A., \& Burki, G. 1998, A\&AS, 129,289

Napiwotzki, R., Shonberner, D., \& Wenske, V. 1993, A\&A, 268, 653

Neiner, C., Hubert, A., \& Catala, C. 2005, ApJS, 156, 237

Netopil, M., Paunzen, E., Maitzen, H. M., North, P., \& Hubrig, S. 2008, A\&A, 491, 545

Paunzen, E., Iliev, I., Kamp, I., \& Barzova, I. 2002, MNRAS, 336, 1030

Popovic, L. C., Simic, S., Milovanovic, N., \& Dimitrijevic, M. 2001, ApJS, 135 , 109

Porter, J. M., \& Rivinius, T. 2003, PASP, 115, 1153

Preston, G. W. 1974, ARA\&A, 12, 257

Przybilla, N., Butler, K., Becker, S. R., \& Kudritzki, R. P. 2001, A\&A, 369, 1009 
C. Saffe and H. Levato: On the nature of sn stars. I.

Przybilla, N., Nieva, M. F., \& Butler, K. 2011, J. Phys. Conf. Ser., 328, 012015 Renson, P., \& Manfroid, J. 2009, A\&A, 498, 961

Richer, J. Michaud, G., \& Turcotte, S. 2000, AJ, 529, 338

Richard, O., Michaud, G., \& Richer, J. 2001, AJ, 558, 377

Rivinius, T., Stefl, S., \& Baade, D. 2006, A\&A, 459, 137

Saffe, C. 2011, Rev. Mex. Astron. Astrofis., 47, 3

Saffe, C., Levato, H., \& López-García, Z. 2005, Rev. Mex. Astron. Astrofis., 41, 415

Saffe, C., Nuñez, N., \& Levato, H. 2011, Rev. Mex. Astron. Astrofis., 47, 219

Shamey, L. 1969, Ph.D. Thesis, University of Colorado at Boulder

Sitnova, T. M., Masonkina, L. I., \& Ryabchikova, T. A. 2013, Astron. Lett., 39, 126

Slettebak, A. 1982, ApJS, 50, 55

Shore, S. N. 1986, ESA SP, 263, 361

Shore, S. N. 1987, AJ, 94, 731
Shore, S. N., \& Adelman, S. J. 1981, in Chemically Peculiar Stars of the Upper Main Sequence, 23rd Liège Astrophysical Symposium (Liège: University of Liège), 429

Shore, S. N., Brown, D. N., \& Sonneborn, G. 1987, AJ, 94, 737

Shore, S. N., Brown, D. N., Sonneborn, G., Landstreet, J., \& Bohlender, D. 1990, AJ, 348, 242

Shore, S. N., Bohlender, D., Bolton, C., North, P., \& Hill, G. M. 2004, A\&A, 421,203

Stauffer, J. R., Barrado y Navascués, D., Bouvier, J., et al. 1999, AJ, 527, 219

Vauclair, S., Dolez, N., \& Gough, D. O. 1991, A\&A, 252, 618

Vick, M., \& Michaud, G. 2008, CoSka, 38, 135

Vick, M., Michaud, G., Richer, J., \& Richard, O. 2010, A\&A, 521, A62

Wolff, S. C., \& Preston, G. W. 1978, ApJS, 37, 371

Zavala, R. T., Adelman, S. J., Hummel, C. A., et al. 2007, AJ, 655, 1046

Zboril, M. 2005, Mem. Soc. Astron. It. Suppl., 7, 136 